\title{
Chemical evolution, petrogenesis, and regional chemical correlations of the flood basalt sequence in the central Deccan Traps, India
}

\author{
L Melluso $^{1 *}$, M Barbieri ${ }^{2}$ and L Beccaluva ${ }^{3}$ \\ ${ }^{1}$ Dipartimento di Scienze della Terra, Università di Napoli Federico II, via Mezzocannone 8, 80134 Napoli, Italy. \\ ${ }^{2}$ Dipartimento di Scienze della Terra, Università di Roma "La Sapienza", Italy. \\ ${ }^{3}$ Dipartimento di Scienze Geologiche, Università di Ferrara, Italy. \\ *e-mail:melluso@unina.it
}

The lava sequence of the central-western Deccan Traps (from Jalgaon towards Mumbai) is formed by basalts and basaltic andesites having a significant variation in $\mathrm{TiO}_{2}$ (from 1.2 to $3.3 \mathrm{wt} \%$ ), $\mathrm{Zr}$ (from 84 to $253 \mathrm{ppm}$ ), $\mathrm{Nb}$ (from 5 to $16 \mathrm{ppm}$ ) and $\mathrm{Ba}$ (from 63 to $407 \mathrm{ppm}$ ), at $\mathrm{MgO}$ ranging from 10 to $4.2 \mathrm{wt} \%$. Most of these basalts follow a liquid line of descent dominated by low pressure fractionation of clinopyroxene, plagioclase and olivine, starting from the most mafic compositions, in a temperature range from $1220^{\circ}$ to $1125^{\circ} \mathrm{C}$. These rocks resemble those belonging to the lowermost formations of the Deccan Traps in the Western Ghats (Jawhar, Igatpuri and Thakurvadi) as well as those of the Poladpur formation. Samples analyzed for ${ }^{87} \mathrm{Sr} /{ }^{86} \mathrm{Sr}$ give a range of initial ratios from 0.70558 to 0.70621 . A group of flows of the Dhule area has low $\mathrm{TiO}_{2}(1.2-1.5 \mathrm{wt} \%)$ and $\mathrm{Zr}(84-105 \mathrm{ppm})$ at moderate $\mathrm{MgO}(5.2-6.2 \mathrm{wt} \%)$, matching the composition of low-Ti basalts of Gujarat, low-Ti dykes of the Tapti swarm and Toranmal basalts, just north of the study area. This allows chemical correlations between the lavas of central Deccan, the Tapti dykes and the northwestern outcrops. The mildly enriched high field strength element contents of the samples with $\mathrm{TiO}_{2}>1.5 \mathrm{wt} \%$ make them products of mantle sources broadly similar to those which generated the Ambenali basalts, but their high $\mathrm{La} / \mathrm{Nb}$ and $\mathrm{Ba} / \mathrm{Nb}$, negative $\mathrm{Nb}$ anomalies in the mantle normalized diagrams, and relatively high ${ }^{87} \mathrm{Sr} /{ }^{86} \mathrm{Sr}$, make evident a crustal input with crustally derived materials at less differentiated stages than those represented in this sample set, or even within the sub-Indian lithospheric mantle.

\section{Introduction}

The Deccan Traps of Western India are one of the best known flood basalt provinces in the world. They form a huge lava field, with associated dyke swarms and intrusive complexes, which cover large parts of peninsular India (figure 1). The rocks were emplaced in a time span from 69 to $63 \mathrm{Ma}$, straddling the Cretaceous-Paleogene boundary, with the most intense volcanic pulse at $66.9 \pm 0.2 \mathrm{Ma}$ (Sen 2001; Mahoney et al 2002; cf. Pande 2002, references therein and subsequent comments). Its reconstructed stratigraphy includes some $2500 \mathrm{~m}$ of mafic lava flows, grouped into eleven formations and three subgroups (the lowermost Kalsubai subgroup, comprising the Igatpuri, Jawhar, Neral, Thakurvadi, and Bhimashankar formations, the Lonavala subgroup, comprising the Khandala and Bushe formations, and the uppermost Wai subgroup, comprising the Poladpur, Ambenali, Mahableshwar, and Panhala formations; cf. Mahoney 1988; Peng et al 1994). The stratigraphy of the Deccan has been first defined in the Western Ghats sections (cf. Najafi et al 1981; Cox and Hawkesworth

Keywords. Petrogenesis; basalts; basaltic andesites; central Deccan Traps; India. 


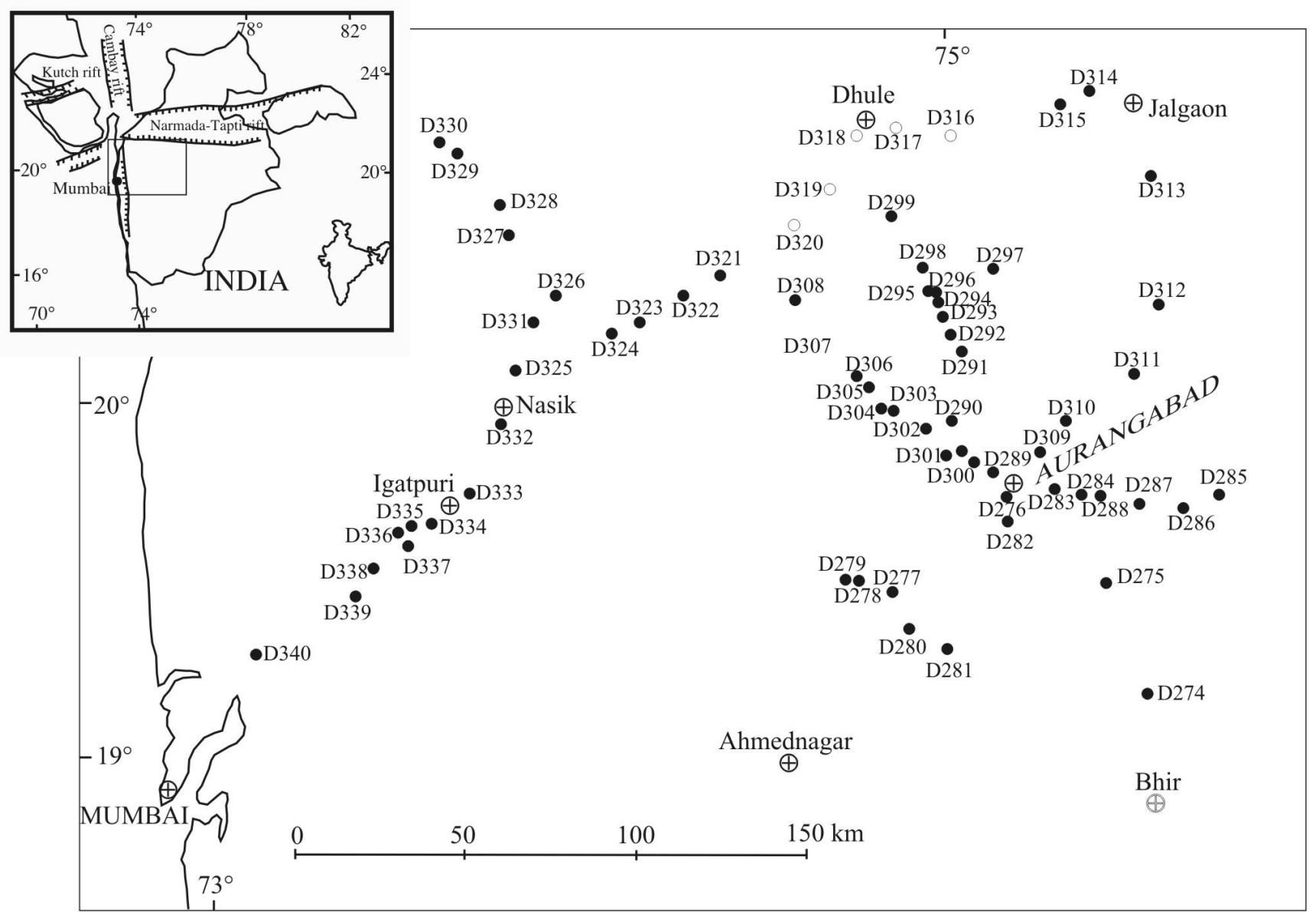

Figure 1. Geological map of central Deccan with location of the samples. The inset shows outcrops of the Deccan Traps. Filled circles: samples with $\mathrm{TiO}_{2}>1.5 \mathrm{wt} \%$; open circles: samples with $\mathrm{TiO}_{2}<1.5 \mathrm{wt} \%$.

1985; Subbarao and Hooper 1988). Several other papers attempted to recognize the known formations elsewhere in the province, often using multielemental statistics (e.g., Mitchell and Widdowson 1991; Peng et al 1998; Khadri et al 1999a, 1999b). Mahoney et al (2000) demonstrated that the lava flows in the northern part of the Deccan, though having generally similar geochemical features with respect to some cropping out in the Western Ghats, crop out with a different stratigraphic sequence (Bushe Fm. upwards, Ambenali and Poladpur intercalated, and downwards Bushe Fm. again; cf. also figure 11 of Widdowson et al 2000, for a general overview). This means that the chemical formations cannot be used as chronostratigraphic markers throughout the province. They could have been erupted from different feeding systems at different times, not resolvable with current radiometric techniques (Peng et al 1998; Hooper 1999; Mahoney et al 2000, 2002).

Further interest to the knowledge of the genesis and the evolution of the Deccan Traps derives from the possibility of correlation between the chemistry of the lava flows cropping out in the
Narmada-Tapti regions and the dyke swarm still extensively exposed (e.g., Melluso et al 1999; Chandrasekharam et al 1999; Mahoney et al 2000; Sheth et al 2004), which may be the source of at least some northern Deccan basalts. The chemical characteristics of the lava flows in the central Deccan, and the relationships between these lavas and the other parts of the Deccan is also an open argument. The lava sequence around Aurangabad is, curiously, poorly known, one of the few published papers being Subbarao et al (1994). This paper is a petrological and geochemical study of an extensive sampling of basalts of central-western Deccan. Particular emphasis will be given to the chemical correlations between the lavas of this area of Deccan and the Tapti dykes, the lavas of TaptiSatpuras and Gujarat.

The Deccan Traps lava flows, though being tholeiitic basalts for the most part, have very heterogeneous geochemical characteristics. The Ambenali basalts have probably the weakest evidence of interaction with continental crust or lithospheric mantle, given their transitional mid-ocean-ridge basalt (T-MORB)-like chemical 
features (e.g., Lightfoot et al 1990). The enriched basalts of Gujarat have most chemical and isotopic features almost identical to the presentday la Reunion basalts (Melluso et al 1995; Peng and Mahoney 1995; J J Mahoney and L Melluso, unpublished data). On the other hand, several formations from the Western Ghats and elsewhere show chemical evidence of interaction with continental crust or lithospheric mantle, from the Bushe $\mathrm{Fm}$. extreme (the lavas of this formation have some of the highest initial ${ }^{87} \mathrm{Sr} /{ }^{86} \mathrm{Sr}$ (up to 0.7200 ) and present-day ${ }^{206} \mathrm{~Pb} /{ }^{204} \mathrm{~Pb}$ (up to 22.46 ) and the lowest initial ${ }^{143} \mathrm{Nd} /{ }^{144} \mathrm{Nd}$ (down to 0.5118 ) ratios, coupled with low $\mathrm{Nb}, \mathrm{Ti}$ and $\mathrm{Zr}$ contents) to the lowermost formations (Peng et al 1994). Peng et al (1994) modelled the crustal inprint with at least two different steps of contamination, characterized by different crustal end-members, which mixed in variable amounts to Ambenali-like mantle-derived magmas. It is worth remembering that major chemical types found in the Deccan have no obvious equivalents in the Western Ghats lava pile. Notable are the highly enriched, and sometimes potassium-rich, basalts cropping out in the Kathiawar peninsula, at Pavagadh, and in the Narmada valley (Krishnamurthy and Cox 1977, 1980; Mahoney et al 1985; Melluso et al 1995), and the highly incompatible element-depleted tholeiites of Gujarat (Melluso et al 1995).

The role of partial melting and mantle source heterogeneity in the petrogenesis of the Deccan Trap basalts, as opposed to the crustal influx, is still very poorly constrained. The magmatic evolution, as well as the petrogenesis of highly evolved rocks like rhyolites, are also an argument open to future modern modelling and debate.

\section{Sampling locations and geological setting}

The samples pertinent to this study have been sampled in road sections from an area comprised within Bhir, Jalgaon, Dhule, the Gujarat state and Mumbai (figure 1). In the area just south of Bhir, flows belonging to Bhimashankar, Khandala, Poladpur, Ambenali and, less frequently, Mahableshwar Fm. have been found (L Melluso, unpublished data; Khadri et al 1999a). Talusani (2001) recently described alkali basalt flows in the area of Bhir. The lava flows have been sampled at different heights, ranging from ca. $860 \mathrm{mt}$, sample D327 (northern tip of the Western Ghats), to Thana, close to the sea level, and to the samples around Dhule (ca. $300 \mathrm{mt}$ a.s.l.). The samples from D328 to D330 have been collected in the Gujarat state. According to the geological map of Subbarao and Hooper (1988), the southwestern-most samples of this study belong to the Igatpuri formation (D340 to D336, from Thana to Igatpuri), whereas the lavas around Nasik belong to the Thakurvadi and Khandala formations (D325 to D335). Subbarao et al (1994) argued that the area of Aurangabad is mostly formed by flows of the lowermost formations (Jawhar and Igatpuri).

\section{Petrography and mineral chemistry}

The sampled lava flows of central Deccan are tholeiitic basalts (72\%) and basaltic andesites (28\%) (figure 2; table 1), with the latter being more abundant at low $\mathrm{MgO}$ contents. They are variably fresh, and porphyritic for phenocrysts of plagioclase, clinopyroxene and less abundant and often altered olivine, with microcrystalline groundmass of variable size, with plagioclase, pyroxenes and more abundant oxides. Mg-rich, olivine-phyric, basalts are not widespread (samples D333 and D325). Plagioclase phenocrysts up to $0.5 \mathrm{~cm}$ in size, have been observed in many samples (D283 to D288, D292 to D295, D334 to D338).

Mineral chemistry: Chemical analyses of the main mineral phases found in a sample subset are given in table 2. Ca-rich clinopyroxene belongs to the augite-ferroaugite (subcalcic ferroaugite) series. Their $\mathrm{Mg} \#$ (atomic $\mathrm{Mg} /(\mathrm{Mg}+\mathrm{Fe})$ ) varies from 0.80 to 0.33 . The $\mathrm{TiO}_{2}$ content of Ca-rich clinopyroxene is low, and ranges from 0.42 to $1.54 \mathrm{wt} \% . \mathrm{Al}_{2} \mathrm{O}_{3}$ is also low and ranges from 0.90 to $2.75 \mathrm{wt} \%$. A few pigeonites have been analysed in sample D316 (table 2). The observed clinopyroxene trend is typical of tholeiitic series magmas. Plagioclase ranges from $\mathrm{An}_{72}$ to $\mathrm{An}_{34}$. Olivine is present as fresh crystals only in a few samples, and ranges

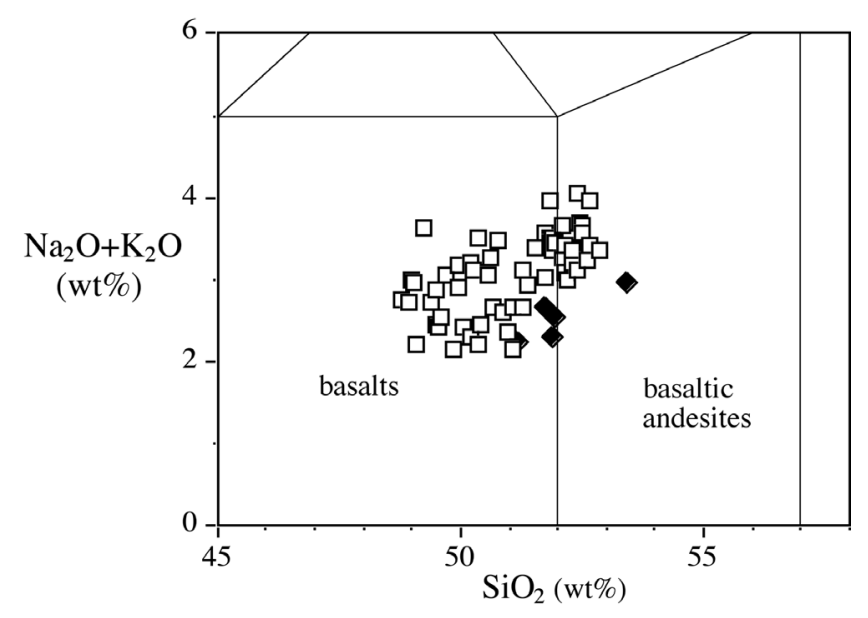

Figure 2. T.A.S. classification diagram for the central Deccan samples. Symbols: open squares: samples with $\mathrm{TiO}_{2}>1.5 \mathrm{wt} \%$; filled diamonds: samples with $\mathrm{TiO}_{2}<1.5$ wt $\%$. 


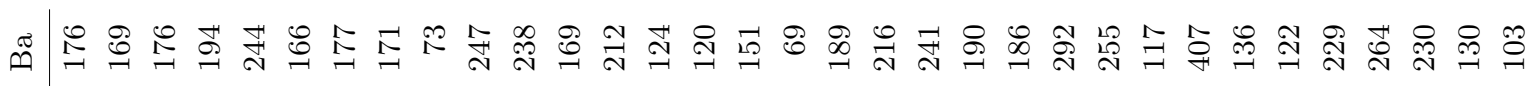

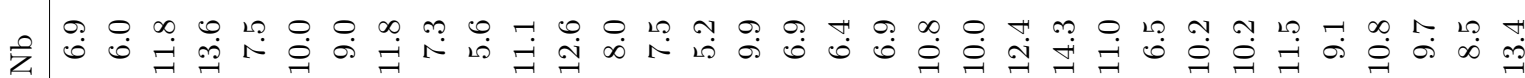

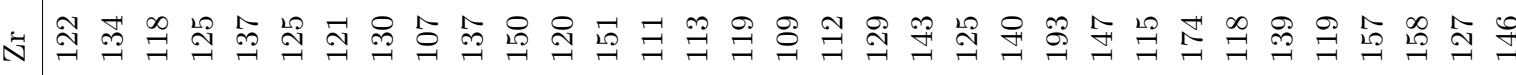

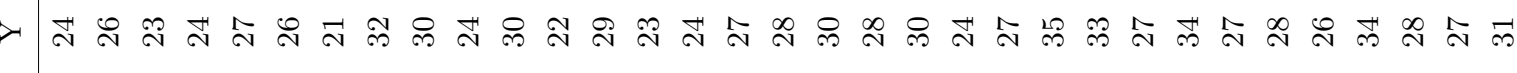

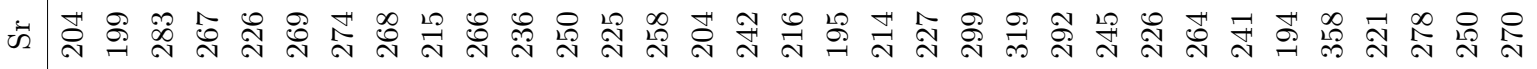

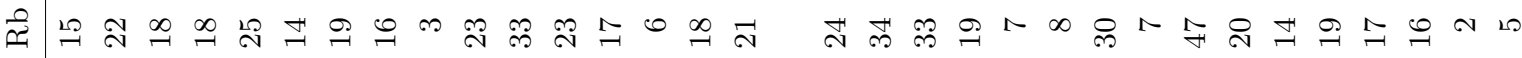
고 苛

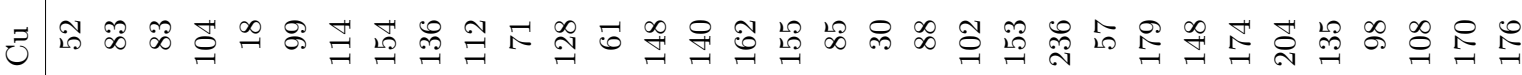

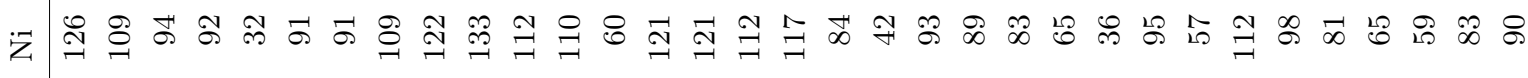

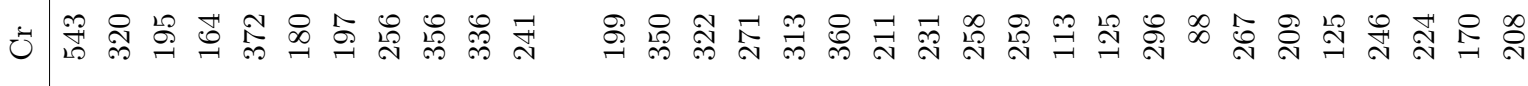

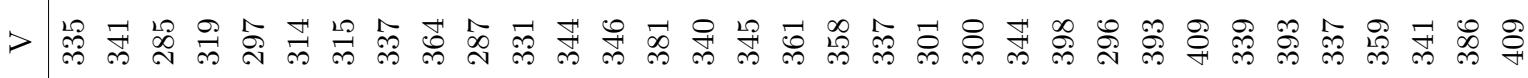

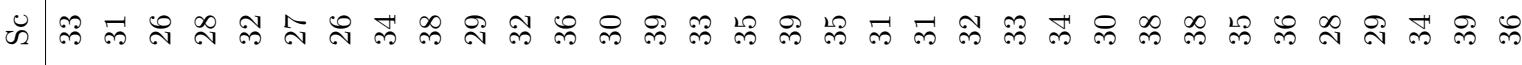

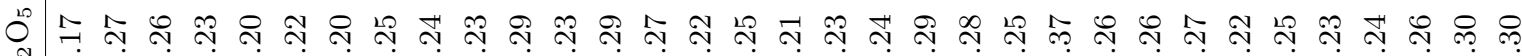

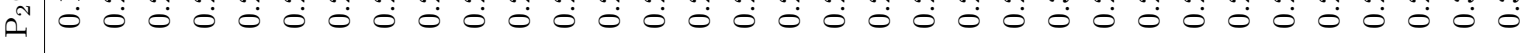

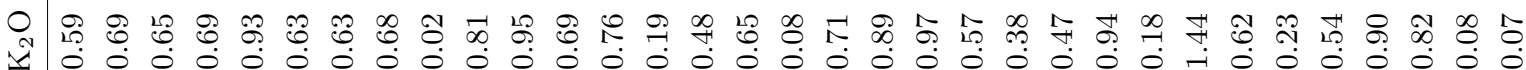

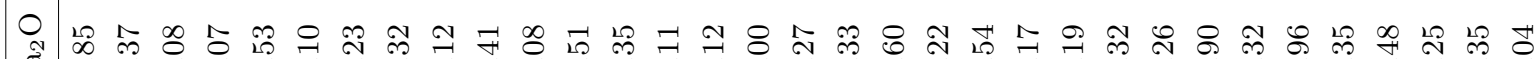

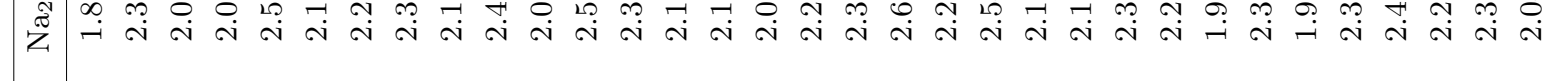
○ \&

임 $\stackrel{\overbrace{}}{\leftrightarrows}$

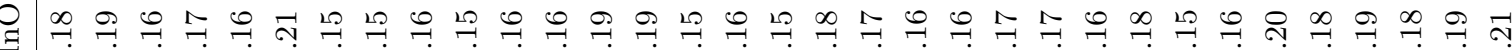

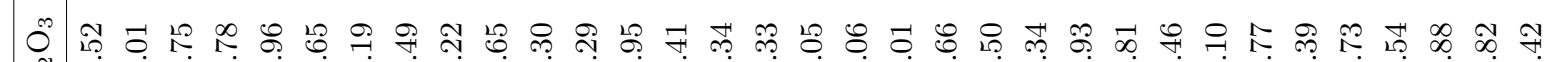


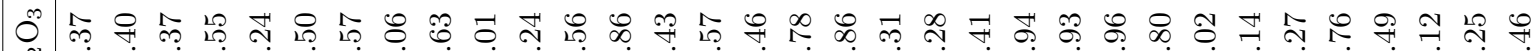

인유

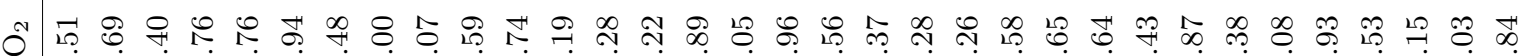

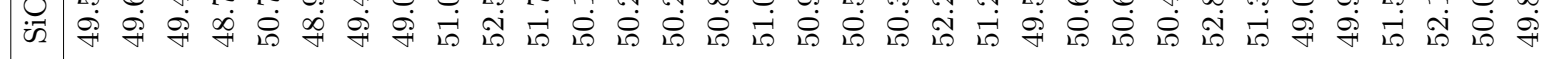




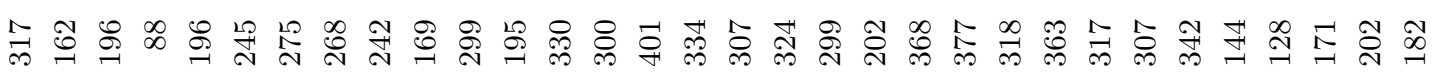
H.

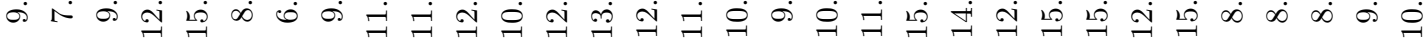

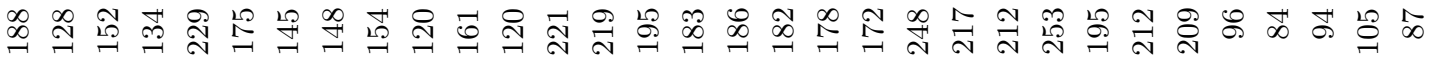
దิ

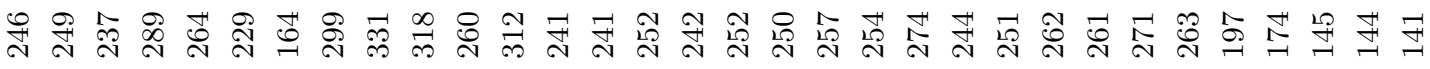
ㄱำ ลำ

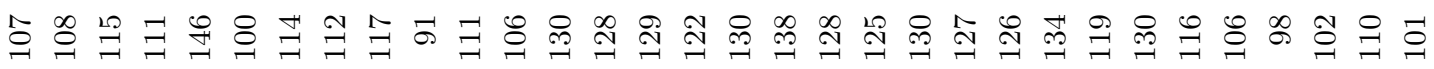

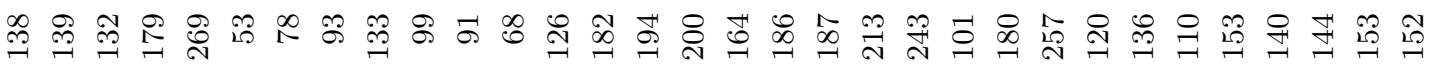

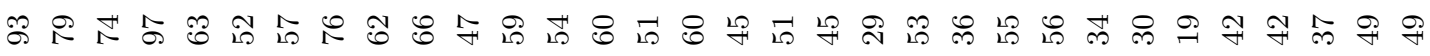

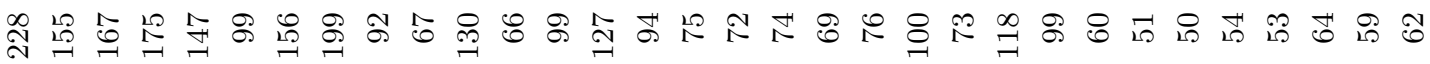

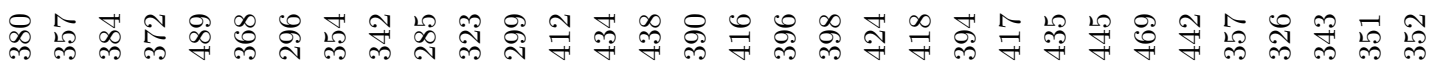
๙ิ

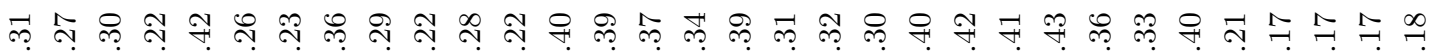

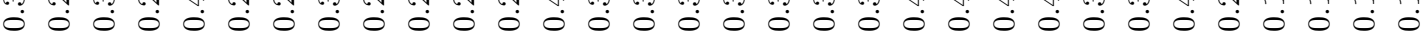
\&

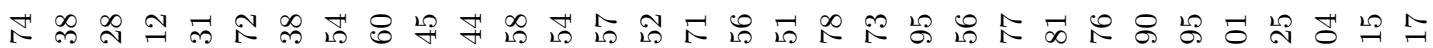

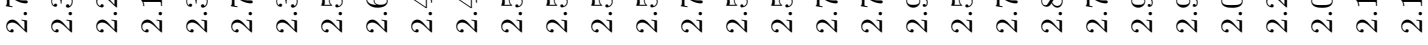
望

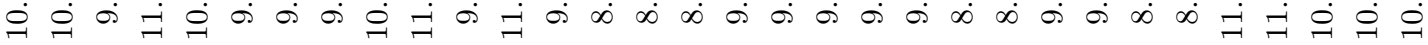
ㄴ ㄱ 구 우

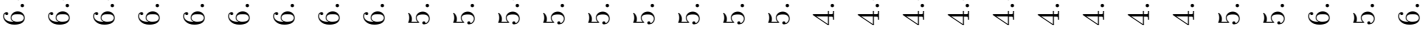

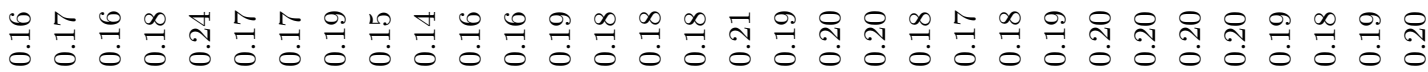

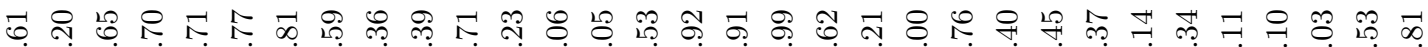

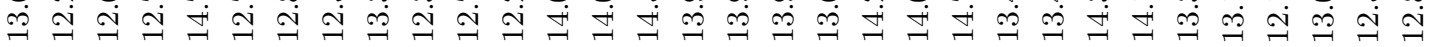

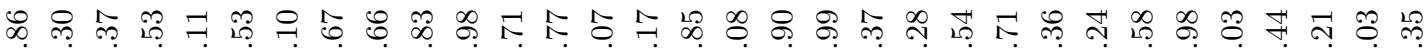

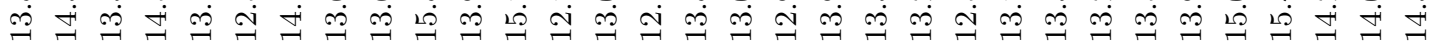
군 뭉 ヘ

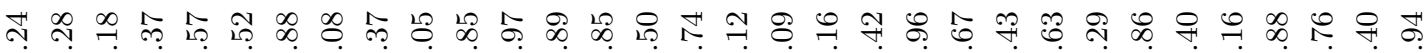
将

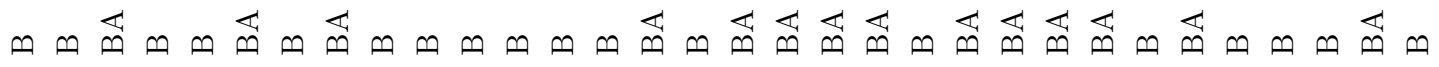

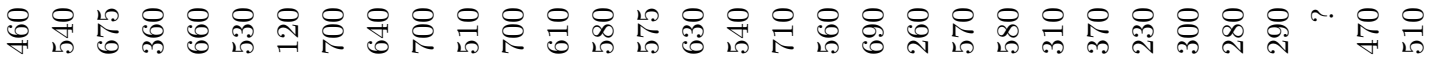


Table 2. Representative analyses of mineral phases in the central Deccan rocks. An, Or, Ab, in mol\%, Ca, Mg, Fe $+\mathrm{Mn}$ in mol\%, ilm and ulv in mole fraction.

\begin{tabular}{|c|c|c|c|c|c|c|c|c|c|c|c|c|c|c|c|c|}
\hline PLAG & & $\mathrm{SiO}_{2}$ & $\mathrm{TiO}_{2}$ & $\mathrm{Al}_{2} \mathrm{O}_{3}$ & $\mathrm{FeO}$ & $\mathrm{MnO}$ & $\mathrm{MgO}$ & $\mathrm{CaO}$ & $\mathrm{Na}_{2} \mathrm{O}$ & $\mathrm{K}_{2} \mathrm{O}$ & $\mathrm{Cr}_{2} \mathrm{O}_{3}$ & $\mathrm{NiO}$ & Sum & An & $\mathrm{Ab}$ & $\mathrm{Or}$ \\
\hline D325 & 1 & 56.15 & & 27.08 & 0.86 & & & 9.61 & 5.56 & 0.53 & & & 99.8 & 47.3 & 49.6 & 3.1 \\
\hline D325 & 1 & 54.22 & & 28.09 & 0.90 & & & 11.19 & 4.98 & 0.36 & & & 99.7 & 54.2 & 43.7 & 2.1 \\
\hline D325 & 1 & 51.02 & & 30.63 & 0.81 & & & 13.65 & 3.50 & 0.17 & & & 99.8 & 67.6 & 31.4 & 1.0 \\
\hline D325 & e & 51.60 & & 30.61 & 0.59 & & & 14.00 & 3.28 & 0.15 & & & 100.2 & 69.6 & 29.5 & 0.9 \\
\hline D333 & 1 & 50.26 & & 30.08 & 0.63 & & & 13.85 & 3.24 & 0.21 & & & 98.3 & 69.4 & 29.4 & 1.3 \\
\hline D333 & 1 & 52.33 & & 28.78 & 1.18 & & & 12.03 & 4.37 & 0.31 & & & 99.0 & 59.2 & 38.9 & 1.8 \\
\hline D316 & $\mathrm{e}$ & 48.14 & & 32.68 & 0.70 & & & 15.96 & 2.08 & 0.07 & & & 99.6 & 80.6 & 19.0 & 0.4 \\
\hline D316 & 1 & 55.93 & & 26.50 & 0.68 & & & 9.65 & 5.48 & 0.33 & & & 98.6 & 48.3 & 49.7 & 2.0 \\
\hline D333 & l & 56.98 & & 26.78 & 0.78 & & & 9.52 & 5.49 & 0.04 & & & 99.6 & 48.8 & 50.9 & 0.2 \\
\hline D293 & $\mathrm{e}$ & 50.37 & & 31.14 & 0.42 & & & 14.73 & 2.98 & 0.18 & & & 99.8 & 72.4 & 26.5 & 1.1 \\
\hline D293 & 1 & 56.83 & & 26.01 & 0.75 & & & 8.93 & 5.89 & 0.63 & & & 99.0 & 43.9 & 52.4 & 3.7 \\
\hline D335 & 1 & 54.84 & & 27.56 & 0.79 & & & 10.30 & 4.82 & 0.50 & & & 98.8 & 52.5 & 44.5 & 3.0 \\
\hline D335 & 1 & 57.57 & & 26.02 & 0.92 & & & 8.42 & 6.11 & 0.64 & & & 99.7 & 41.6 & 54.6 & 3.8 \\
\hline D335 & e & 51.90 & & 30.42 & 0.58 & & & 13.46 & 3.65 & 0.25 & & & 100.3 & 66.1 & 32.4 & 1.5 \\
\hline D335 & 1 & 59.67 & & 24.41 & 0.56 & & & 6.75 & 6.78 & 0.91 & & & 99.1 & 33.6 & 61.0 & 5.4 \\
\hline PX & & & & & & & & & & & & & & $\mathrm{Ca}$ & $\mathbf{F e}^{*}$ & $\mathrm{Mg}$ \\
\hline D325 & e & 52.27 & 0.69 & 2.69 & 7.35 & 0.09 & 16.67 & 19.31 & 0.28 & & 0.63 & & 100.0 & 40.0 & 12.0 & 48.0 \\
\hline D325 & 1 & 51.75 & 1.12 & 2.47 & 9.64 & 0.22 & 15.43 & 19.18 & 0.22 & & 0.29 & & 100.3 & 39.7 & 15.9 & 44.4 \\
\hline D325 & e & 52.66 & 0.87 & 2.12 & 8.03 & 0.08 & 16.34 & 19.89 & 0.28 & & 0.44 & & 100.7 & 40.6 & 12.9 & 46.4 \\
\hline D325 & 1 & 51.83 & 0.96 & 1.27 & 13.92 & 0.41 & 14.35 & 16.93 & 0.28 & & 0.00 & & 100.0 & 35.2 & 23.3 & 41.5 \\
\hline D325 & l & 53.64 & 0.38 & 0.38 & 15.90 & 0.39 & 11.83 & 18.62 & 0.19 & & 0.00 & & 101.3 & 39.0 & 26.6 & 34.4 \\
\hline D333 & 1 & 52.49 & 0.68 & 2.18 & 7.37 & 0.29 & 16.29 & 19.86 & 0.21 & & 0.30 & & 99.7 & 40.9 & 12.3 & 46.7 \\
\hline D333 & 1 & 51.72 & 0.94 & 2.47 & 8.80 & 0.30 & 16.26 & 18.44 & 0.26 & & 0.43 & & 99.6 & 38.3 & 14.8 & 47.0 \\
\hline D316 & $\mathrm{e}$ & 52.17 & 0.43 & 2.15 & 8.24 & 0.18 & 16.67 & 18.51 & 0.18 & & 0.10 & & 98.6 & 38.3 & 13.6 & 48.0 \\
\hline D316 & 1 & 50.31 & 0.97 & 2.01 & 16.83 & 0.36 & 13.00 & 15.85 & 0.27 & & 0.00 & & 99.6 & 33.5 & 28.3 & 38.2 \\
\hline D316 & 1 & 50.75 & 0.68 & 1.13 & 23.22 & 0.62 & 12.37 & 11.24 & 0.18 & & 0.00 & & 100.2 & 23.9 & 39.6 & 36.6 \\
\hline D316 & l & 51.69 & 0.44 & 1.43 & 18.62 & 0.43 & 18.61 & 7.56 & 0.11 & & 0.00 & & 98.9 & 15.6 & 30.8 & 53.6 \\
\hline D316 & 1 & 53.03 & 0.29 & 0.70 & 20.11 & 0.54 & 21.20 & 3.98 & 0.06 & & 0.00 & & 99.9 & 8.0 & 32.5 & 59.5 \\
\hline D316 & 1 & 49.41 & 0.42 & 0.74 & 29.99 & 0.81 & 8.58 & 10.18 & 0.13 & & 0.00 & & 100.3 & 22.1 & 52.1 & 25.9 \\
\hline D293 & 1 & 49.98 & 1.37 & 0.93 & 20.38 & 0.64 & 10.28 & 15.75 & 0.27 & & 0.00 & & 99.6 & 33.9 & 35.3 & 30.8 \\
\hline D293 & 1 & 50.88 & 1.24 & 1.93 & 11.75 & 0.33 & 14.72 & 18.56 & 0.23 & & 0.00 & & 99.6 & 38.3 & 19.5 & 42.2 \\
\hline D293 & 1 & 51.42 & 1.08 & 2.58 & 10.11 & 0.20 & 14.85 & 19.62 & 0.20 & & 0.00 & & 100.1 & 40.6 & 16.7 & 42.7 \\
\hline D293 & $\mathrm{e}$ & 51.52 & 1.08 & 2.13 & 9.46 & 0.28 & 14.77 & 19.80 & 0.23 & & 0.13 & & 99.4 & 41.3 & 15.9 & 42.9 \\
\hline D293 & 1 & 50.02 & 1.54 & 1.35 & 16.57 & 0.50 & 11.01 & 18.04 & 0.29 & & 0.00 & & 99.3 & 38.6 & 28.5 & 32.8 \\
\hline D335 & $\mathrm{e}$ & 51.00 & 1.21 & 2.66 & 10.82 & 0.27 & 14.09 & 19.06 & 0.28 & & 0.54 & & 99.9 & 40.3 & 18.3 & 41.4 \\
\hline D335 & 1 & 51.59 & 1.09 & 2.24 & 9.39 & 0.27 & 14.73 & 19.93 & 0.26 & & 0.36 & & 99.9 & 41.6 & 15.7 & 42.7 \\
\hline D335 & l & 50.99 & 0.92 & 0.90 & 17.02 & 0.56 & 11.25 & 17.89 & 0.26 & & 0.07 & & 99.9 & 37.8 & 29.0 & 33.1 \\
\hline OLIV & & $\mathrm{SiO}_{2}$ & $\mathrm{TiO}_{2}$ & $\mathrm{Al}_{2} \mathrm{O}_{3}$ & $\mathrm{FeO}$ & $\mathrm{MnO}$ & $\mathrm{MgO}$ & $\mathrm{CaO}$ & $\mathrm{Na}_{2} \mathrm{O}$ & $\mathrm{K}_{2} \mathrm{O}$ & $\mathrm{Cr}_{2} \mathrm{O}_{3}$ & $\mathrm{NiO}$ & & $\mathrm{Mg} \#$ & & \\
\hline D325 & 1 & 34.27 & & & 41.76 & 1.14 & 22.69 & 0.36 & & & & 0.09 & 100.3 & 0.49 & & \\
\hline D325 & 1 & 36.29 & & & 32.21 & 0.85 & 30.32 & 0.25 & & & & 0.13 & 100.1 & 0.62 & & \\
\hline D333 & $\mathrm{e}$ & 38.66 & & & 16.50 & 0.53 & 42.53 & 0.36 & & & & 0.14 & 98.7 & 0.82 & & \\
\hline D333 & l & 37.27 & & & 25.88 & 0.63 & 34.90 & 0.30 & & & & 0.10 & 99.1 & 0.70 & & \\
\hline D333 & 1 & 34.28 & & & 39.25 & 0.96 & 23.20 & 0.36 & & & & 0.08 & 98.1 & 0.51 & & \\
\hline D335 & 1 & 34.07 & & & 44.14 & 1.16 & 19.36 & 0.62 & & & & 0.05 & 99.4 & 0.43 & & \\
\hline D335 & 1 & 33.51 & & & 47.94 & 1.40 & 16.58 & 0.38 & & & & 0.03 & 99.8 & 0.37 & & \\
\hline D335 & $\mathrm{e}$ & 37.11 & & & 29.60 & 0.78 & 32.39 & 0.37 & & & & 0.14 & 100.4 & 0.66 & & \\
\hline D335 & $\mathrm{e}$ & 36.75 & & & 32.38 & 0.70 & 30.22 & 0.32 & & & & 0.21 & 100.6 & 0.62 & & \\
\hline D293 & 1 & 34.10 & & & 42.71 & 1.09 & 21.28 & 0.42 & & & & 0.06 & 99.7 & 0.46 & & \\
\hline D293 & 1 & 32.74 & & & 49.64 & 1.43 & 14.72 & 0.47 & & & & 0.08 & 99.1 & 0.34 & & \\
\hline D293 & l & 36.30 & & & 33.07 & 0.85 & 29.26 & 0.40 & & & & 0.12 & 100.0 & 0.61 & & \\
\hline
\end{tabular}


Table 2. (Continued).

\begin{tabular}{|c|c|c|c|c|c|c|c|c|c|c|c|c|c|c|c|}
\hline SPIN & & & & & & & & & & & & & & Ulv & \\
\hline D325 & 1 & & 11.72 & 1.27 & 80.06 & 0.17 & 0.42 & & & & 0.35 & & 94.0 & 0.33 & \\
\hline D333 & $\mathrm{e}$ & & 1.61 & 18.19 & 27.55 & 0.25 & 9.52 & & & & 40.68 & & 97.8 & 0.06 & \\
\hline D333 & 1 & & 15.19 & 1.71 & 74.49 & 0.87 & 0.37 & & & & 0.14 & & 92.8 & 0.38 & \\
\hline D316 & 1 & & 25.78 & 2.12 & 61.32 & 0.97 & 0.46 & & & & 0.00 & & 90.7 & 0.46 & \\
\hline D293 & 1 & & 34.51 & 1.05 & 57.68 & 0.77 & 1.35 & & & & 0.07 & & 95.4 & & \\
\hline D293 & 1 & & 28.77 & 1.06 & 63.04 & 1.77 & 0.49 & & & & 0.00 & & 95.1 & 0.48 & \\
\hline D293 & 1 & & 22.82 & 1.81 & 67.42 & 0.97 & 0.55 & & & & 0.07 & & 93.6 & 0.44 & \\
\hline D335 & 1 & & 26.23 & 1.71 & 64.90 & 1.40 & 0.09 & & & & 0.29 & & 94.6 & 0.46 & \\
\hline ILM & & & & & & & & & & & & & & & Ilm \\
\hline D325 & 1 & & 49.57 & 0.00 & 47.91 & 0.56 & 1.06 & & & & 0.17 & & 99.3 & & 0.94 \\
\hline D325 & 1 & & 49.09 & 0.05 & 47.50 & 0.40 & 1.33 & & & & 0.22 & & 98.6 & & 0.93 \\
\hline D325 & 1 & & 50.95 & 0.16 & 46.31 & 0.38 & 2.07 & & & & 0.11 & & 100.0 & & 0.95 \\
\hline D333 & 1 & & 48.89 & 0.03 & 47.42 & 0.44 & 1.26 & & & & 0.08 & & 98.1 & & 0.93 \\
\hline D293 & 1 & & 50.92 & 0.25 & 42.23 & 0.57 & 1.16 & & & & 0.13 & & 95.3 & & 1.00 \\
\hline AMPH & & & & & & & & & & & $\mathrm{F}$ & $\mathrm{Cl}$ & & & \\
\hline D325 & 1 & 48.82 & 1.21 & 4.67 & 14.88 & 0.13 & 14.45 & 10.58 & 2.09 & 0.80 & 0.15 & 2.78 & 100.6 & & \\
\hline D325 & 1 & 49.01 & 1.01 & 4.79 & 14.65 & 0.24 & 14.70 & 10.38 & 1.91 & 0.67 & 0.28 & 2.54 & 100.2 & & \\
\hline
\end{tabular}

$\mathrm{Mg} \#=$ atomic $\mathrm{Mg}^{*} 100 /(\mathrm{Mg}+\mathrm{Fe})$.

e $=$ early.

$1=$ late.

in composition from $\mathrm{Fo}_{82}$ (cores of sample D333) to $\mathrm{Fo}_{34}$ (sample D293). It is often included in plagioclase; more rarely the reverse is observed. The relatively Fe-rich nature of the analyses is remarked. Spinels are chromiferous when included in $\mathrm{Mg}$ olivine $\left(\mathrm{Cr}_{2} \mathrm{O}_{3}\right.$ up to 40.7 wt.\% in D333). Groundmass titanomagnetites are Al-poor $(0.35-2.12 \mathrm{wt} \%$ as oxide). Ilmenites are Mn-poor (0.38-0.57 wt\% as $\mathrm{MnO}$ ). Rare Fe-hornblende has been found in the interstices of sample D325.

\section{Geochemistry of the lava flows}

The lava flows of the central-western Deccan have a wide range of $\mathrm{MgO}$ (from 10 to $4.2 \mathrm{wt} \%$ ), $\mathrm{TiO}_{2}$ (from 1.2 to $3.3 \mathrm{wt} \%$ ) at $\mathrm{SiO}_{2}$ from 48 to $53 \mathrm{wt} \%$. Their $\mathrm{Mg} /\left(\mathrm{Mg}+\mathrm{Fe}^{2+}\right)$ ratio ranges from 0.65 to 0.40. The sample D333 has characteristics similar to primitive magmas, and also its most forsteriterich olivine we have found $\left(\mathrm{Fo}_{82}\right)$ is close to that expected from chemical equilibrium with the host rock $\left(\mathrm{Fo}_{85}\right)$ (cf. also Beane and Hooper 1988, for further discussion on Mg-rich samples of this area of the Western Ghats). The concentration of incompatible trace elements is widely variable (e.g., $\mathrm{Zr}$ from 84 to $250 \mathrm{ppm}$; Y from 23 to $45 \mathrm{ppm}$; Nb from 6 to $16 \mathrm{ppm}) . \mathrm{K}_{2} \mathrm{O}, \mathrm{Rb}$ and $\mathrm{Ba}$ reach values as high as $1.44 \mathrm{wt} \%, 47 \mathrm{ppm}$ and $407 \mathrm{ppm}$, respectively, in basaltic andesites found north of the Bhir village (D274, D275).
Representative major and trace element diagrams, using $\mathrm{Zr}$ as differentiation index (Hooper 1994), are shown in figure 4 . The samples show a trend of $\mathrm{CaO}, \mathrm{MgO}, \mathrm{Cr}$ and $\mathrm{Ni}$ decrease with increasing $\mathrm{Zr}$, whereas $\mathrm{Ba}, \mathrm{K}_{2} \mathrm{O}, \mathrm{V}, \mathrm{Zn}$, $\mathrm{Y}, \mathrm{Nb}$ and $\mathrm{TiO}_{2}$ contents increase. However, plagioclase-phyric basaltic and basaltic andesite lava flows occurring in the neighborhoods of Dhule (D316 to D320, figure 1, table 1) have peculiar chemical characteristics, with respect to the bulk of the lava flows of the central Deccan: at moderate $\mathrm{MgO}$ (5-6.2 $\mathrm{wt} \%$ ), they have low $\mathrm{TiO}_{2}$ $(1.2-1.5 \mathrm{wt} \%)$ and $\mathrm{Zr}(<105 \mathrm{ppm})$. They have also relatively high $\mathrm{Sc}$ contents $(37-43 \mathrm{ppm})$ and $\mathrm{Al}_{2} \mathrm{O}_{3}$ (14-15.4 wt\%). These samples plot clearly outside the trends depicted from the other samples (figures 2, 4).

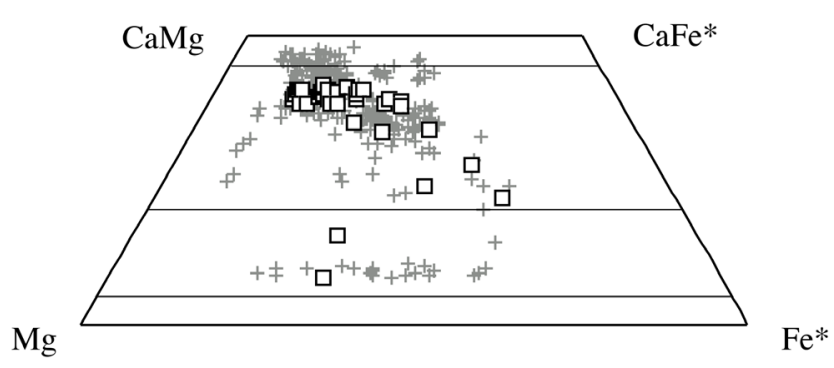

Figure 3. Pyroxene diagram for the central-western Deccan samples (squares). The pyroxenes of the Gujarat tholeiitic basalts (Melluso et al 1995) are shown in grey for comparison. 

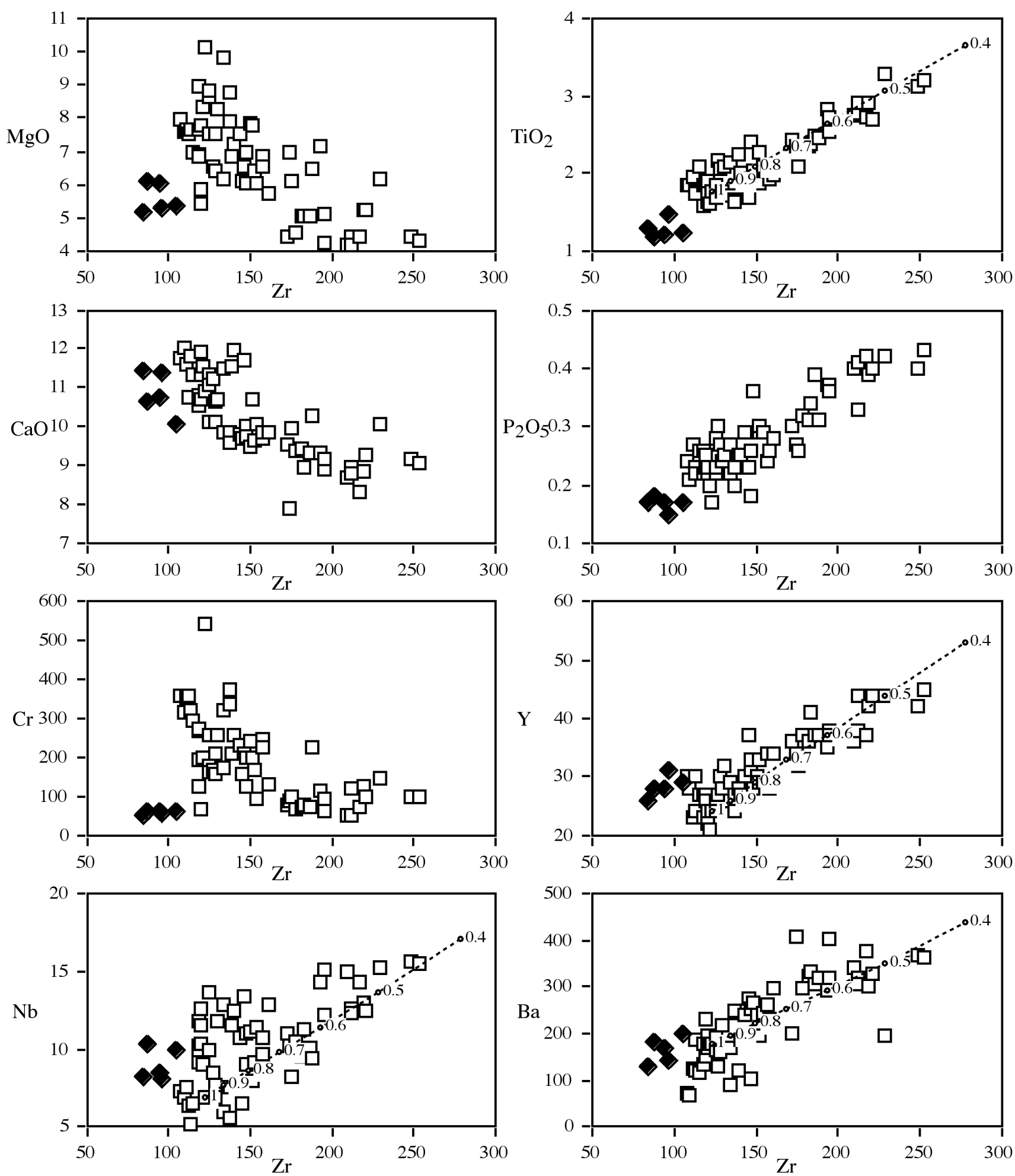

Figure 4. Representative variation diagrams using $\mathrm{Zr}$ as differentiation index. Rayleigh fractional crystallization modelling from sample D333 is reported in a few diagrams. Bulk-rock partition coefficients used: $\mathrm{D}_{\mathrm{Zr}}=0.1 ; \mathrm{D}_{\mathrm{Nb}}$ and $\mathrm{D}_{\mathrm{Ba}}=0.01$; $\mathrm{D}_{\mathrm{Ti}}=0.2 ; \mathrm{D}_{\mathrm{Y}}=0.14$ (cf. Melluso et al 2001). Symbols as in figure 2.

The central-western Deccan samples span a moderate range of $\mathrm{Zr} / \mathrm{Y}(3.1-5.9)$ and $\mathrm{Ti} / \mathrm{V}$ (20-44), with the lowest values found in the samples with low $\mathrm{TiO}_{2}(\mathrm{Zr} / \mathrm{Y}=3.1-3.6$ and
$\mathrm{Ti} / \mathrm{V}=20-25)$. The samples have relatively high $\mathrm{Ba} / \mathrm{Nb}$ and $\mathrm{La} / \mathrm{Nb}(13-40$ and $1.15-2.9$, respectively), and low Nb/U (7-19). There is no correlation between the value of these ratios and degree 
Table 3. Rare earths and other element contents of representative central Deccan samples.

\begin{tabular}{|c|c|c|c|c|c|c|c|c|c|c|c|c|c|c|}
\hline Sample & D333 & D325 & D331 & D274 & D330 & D335 & D339 & D293 & D294 & D288 & D313 & $\begin{array}{c}\text { Low-Ti } \\
\text { D320 }\end{array}$ & $\begin{array}{c}\text { Low-Ti } \\
\text { D319 }\end{array}$ & $\begin{array}{c}\text { Low-Ti } \\
\text { D316 }\end{array}$ \\
\hline La ppm & 12.54 & 13.43 & 21.82 & 22.56 & 15.42 & 18.63 & 18.46 & 13.68 & 15.88 & 25.27 & 27.73 & 12.40 & 16.32 & 11.90 \\
\hline $\mathrm{Ce}$ & 27.95 & 30.24 & 49.31 & 50.03 & 34.69 & 41.62 & 39.65 & 31.55 & 36.85 & 58.94 & 63.99 & 24.71 & 33.03 & 25.46 \\
\hline $\operatorname{Pr}$ & 3.74 & 4.18 & 6.60 & 6.65 & 4.61 & 5.41 & 5.21 & 4.14 & 5.01 & 8.06 & 8.65 & 3.05 & 4.12 & 3.18 \\
\hline $\mathrm{Nd}$ & 17.09 & 18.09 & 27.73 & 29.54 & 19.23 & 24.23 & 21.24 & 17.93 & 23.51 & 35.19 & 37.14 & 13.92 & 15.20 & 13.39 \\
\hline $\mathrm{Sm}$ & 4.21 & 4.74 & 7.17 & 7.29 & 4.68 & 6.37 & 5.26 & 4.32 & 5.59 & 8.67 & 8.79 & 3.62 & 3.83 & 3.48 \\
\hline $\mathrm{Eu}$ & 1.48 & 1.38 & 2.00 & 2.23 & 1.54 & 2.13 & 1.54 & 1.51 & 1.68 & 2.46 & 2.49 & 1.14 & 1.18 & 1.13 \\
\hline Gd & 4.98 & 5.12 & 7.75 & 7.18 & 5.02 & 6.57 & 6.02 & 4.21 & 4.97 & 8.98 & 9.04 & 3.58 & 4.82 & 3.92 \\
\hline $\mathrm{Tb}$ & 0.74 & 0.86 & 1.35 & 1.14 & 0.79 & 1.02 & 1.07 & 0.63 & 0.82 & 1.46 & 1.45 & 0.62 & 0.82 & 0.69 \\
\hline Dy & 4.18 & 5.33 & 8.01 & 7.05 & 4.64 & 5.78 & 6.65 & 3.84 & 5.11 & 8.85 & 8.67 & 4.34 & 5.02 & 4.69 \\
\hline Ho & 0.80 & 1.08 & 1.49 & 1.43 & 0.91 & 1.16 & 1.38 & 0.80 & 1.01 & 1.75 & 1.75 & 1.03 & 1.09 & 1.07 \\
\hline Er & 2.01 & 2.82 & 3.82 & 3.71 & 2.43 & 2.93 & 3.57 & 1.94 & 2.59 & 4.56 & 4.50 & 2.75 & 2.98 & 2.62 \\
\hline Tm & 0.30 & 0.40 & 0.51 & 0.50 & 0.33 & 0.44 & 0.53 & 0.28 & 0.33 & 0.63 & 0.63 & 0.39 & 0.46 & 0.41 \\
\hline $\mathrm{Yb}$ & 1.90 & 2.50 & 3.43 & 2.99 & 2.02 & 2.60 & 3.56 & 1.74 & 2.06 & 3.90 & 3.97 & 2.16 & 3.03 & 2.68 \\
\hline $\mathrm{Lu}$ & 0.28 & 0.35 & 0.53 & 0.42 & 0.30 & 0.41 & 0.54 & 0.27 & 0.29 & 0.56 & 0.55 & 0.31 & 0.42 & 0.46 \\
\hline $\mathrm{Hf}$ & 3.26 & 4.55 & 5.21 & 4.76 & 4.34 & 4.74 & 4.64 & 2.82 & 4.40 & 5.36 & 5.53 & 3.98 & 4.15 & 2.49 \\
\hline $\mathrm{Ta}$ & 0.55 & 0.42 & & 0.54 & 0.63 & 0.75 & 0.57 & 0.79 & 0.71 & 0.76 & 0.91 & 0.39 & 0.48 & 0.61 \\
\hline $\mathrm{Pb}$ & 2.04 & & & & & 3.04 & & 1.73 & & & & & & 2.23 \\
\hline $\mathrm{Th}$ & 3.24 & 2.05 & 6.29 & 3.02 & 2.18 & 2.27 & 4.04 & 2.07 & 2.07 & 3.27 & 3.98 & 3.76 & 3.64 & 2.18 \\
\hline $\mathrm{U}$ & 0.73 & 0.53 & 1.14 & 0.55 & 0.56 & 0.42 & 0.93 & 0.51 & 0.55 & 0.71 & 0.90 & 0.83 & 0.78 & 0.54 \\
\hline $\mathrm{La} / \mathrm{Yb}$ & 4.5 & 3.6 & 4.3 & 5.1 & 5.1 & 4.8 & 3.5 & 5.3 & 5.2 & 4.4 & 4.7 & 3.9 & 3.6 & 3.0 \\
\hline $\mathrm{La} / \mathrm{Nd}$ & 1.42 & 1.44 & 1.52 & 1.48 & 1.55 & 1.49 & 1.68 & 1.48 & 1.31 & 1.39 & 1.45 & 1.72 & 2.08 & 1.72 \\
\hline $\mathrm{La} / \mathrm{Sm}$ & 1.87 & 1.78 & 1.92 & 1.95 & 2.07 & 1.84 & 2.21 & 1.99 & 1.79 & 1.83 & 1.99 & 2.15 & 2.68 & 2.15 \\
\hline $\mathrm{Eu} / \mathrm{Eu}^{*}$ & 0.98 & 0.85 & 0.82 & 0.93 & 0.96 & 1.00 & 0.83 & 1.07 & 0.97 & 0.85 & 0.85 & 0.96 & 0.83 & 0.93 \\
\hline
\end{tabular}

Ratios are chondrite normalised.

$\mathrm{Eu} / \mathrm{Eu}^{*}=\mathrm{Eu}_{\mathrm{n}} /\left(\sqrt{ } \mathrm{Gd}_{\mathrm{n}}^{*} \mathrm{Sm}_{\mathrm{n}}\right)$.

of differentiation (e.g., with decreasing $\mathrm{MgO}$ or increasing $\mathrm{Zr}$ contents).

The samples D277 and D279 have low Zr/Y (3.53.8) at $\mathrm{TiO}_{2}=1.84-1.86 \mathrm{wt} \%$, and have also low $\mathrm{Ba} / \mathrm{Y}(2.4)$. They seem to be quite similar to samples of the Ambenali Fm., which is characterized by similar range of these oxides and ratios (cf. Lightfoot et al 1990). The samples D327 and D339 have low $\mathrm{Zr} / \mathrm{Y}$ (3.7-3.9), with high $\mathrm{TiO}_{2}$ (1.70$1.76 \mathrm{wt} \%)$ and $\mathrm{Ba} / \mathrm{Y}(6.3-7.5)$. From their stratigraphic position and chemistry, they should belong to the Thakurvadi or Khandala Fms.

The lantanide rare earth element (REE) contents more than double from the least to the most evolved compositions $(\Sigma \mathrm{REE}=82 \mathrm{ppm}$ in D333; $\Sigma$ REE $=179 \mathrm{ppm}$ in D313; table 3 and figure 5). The low-Ti samples have some of the lowest REE contents ( $\mathrm{REE}=74-92 \mathrm{ppm})$, taking into account their evolved nature. The samples are moderately light rare earth elementenriched $\left((\mathrm{La} / \mathrm{Yb})_{n}\right.$ from 3.0 to 5.1 ; the subscript $n$ means normalized to chondritic values). The heavy rare earth element patterns are substantially flat $\left((\mathrm{Tb} / \mathrm{Yb})_{n}\right.$ from 1.1 to 1.7$)$, with the lowest values found in the low-Ti basalts
$\left((\mathrm{Tb} / \mathrm{Yb})_{n}=1.1-1.3\right)$. Small negative europium anomalies are noted in the evolved samples $\left(\mathrm{Eu} / \mathrm{Eu}^{*}=0.85-0.83\right.$, where $\mathrm{Eu}$ is normalized europium and $\mathrm{Eu}^{*}$ is europium interpolated between normalized samarium and gadolinium).

\section{Sr-isotopes}

Fourteen samples of basalts and basaltic andesites were analyzed for Sr-isotopic composition (table 4). The values recalculated at $66 \mathrm{Ma}$ range from 0.70558 to 0.70618 . The data thus show minor variation, when compared to the very wide range observed in the Deccan Trap lava flows (0.7038 to 0.72315 ; e.g. Peng et al 1994; Chandrasekharam et al 1999). Nevertheless, these data are significantly higher than typical Ambenali basalts $\left({ }^{87} \mathrm{Sr} /{ }^{86} \mathrm{Sr}=0.70383-0.70558\right)$ and also higher than the lowest values of Pavagadh/drillhole samples of Gujarat $\left({ }^{87} \mathrm{Sr} /{ }^{86} \mathrm{Sr}=0.70414-0.70457\right.$; Peng and Mahoney 1995), testifying input of different source components or the effects of increasing ${ }^{87} \mathrm{Sr} /{ }^{86} \mathrm{Sr}$ due to crustal contamination. The data on the other hand, are similar to those obtained 

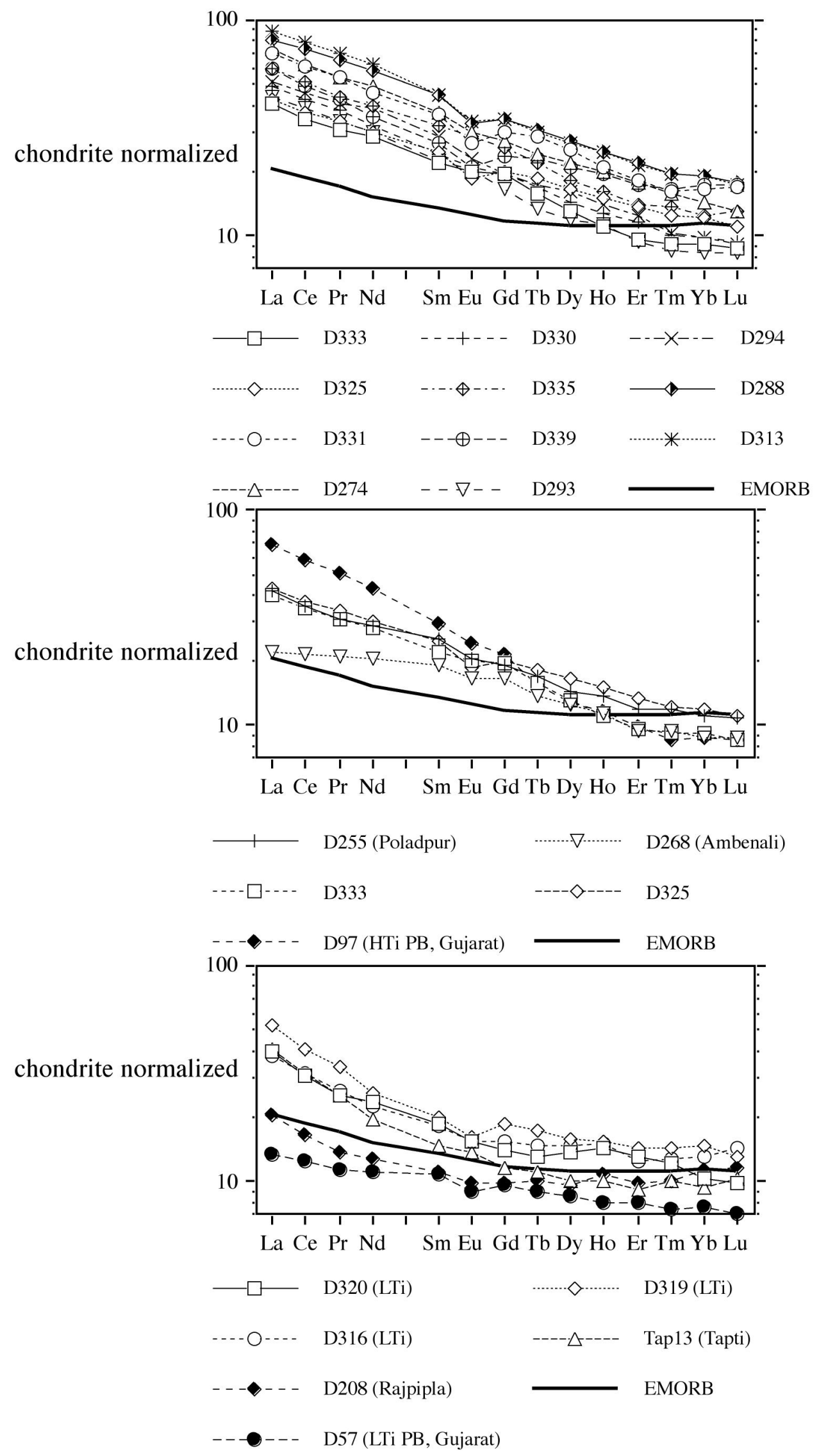

Figure 5. Chondrite normalized rare earth element diagrams for central western Deccan samples. E-MORB composition from Sun and McDonough (1989). Other samples from Melluso et al (1995) or L Melluso (unpublished data). Normalization values of Boynton (1984). 
Table 4. Sr isotopic composition of samples from central Deccan.

\begin{tabular}{|c|c|c|c|c|c|c|}
\hline & \multicolumn{6}{|c|}{$\left({ }^{87} \mathrm{Sr} /{ }^{86} \mathrm{Sr}\right)$} \\
\hline & $\left({ }^{87} \mathrm{Sr} /{ }^{86} \mathrm{Sr}\right) \mathrm{i}$ & $\mathrm{Rb}$ & $\mathrm{Sr}$ & meas & $\mathrm{Rb} / \mathrm{Sr}$ & ${ }^{87} \mathrm{Rb} /{ }^{86} \mathrm{Sr}$ \\
\hline M284 & 0.70621 & 30 & 241 & 0.70655 & 0.124 & 0.360 \\
\hline M286 & 0.70593 & 10 & 257 & 0.70604 & 0.039 & 0.113 \\
\hline M287 & 0.70596 & 25 & 251 & 0.70645 & 0.100 & 0.288 \\
\hline M299 & 0.70558 & 30 & 245 & 0.70591 & 0.122 & 0.354 \\
\hline M300 & 0.70605 & 30 & 263 & 0.70662 & 0.114 & 0.330 \\
\hline M304 & 0.70618 & 20 & 241 & 0.70641 & 0.083 & 0.240 \\
\hline M306 & 0.70611 & 21 & 242 & 0.70635 & 0.087 & 0.251 \\
\hline M307 & 0.70562 & 25 & 226 & 0.70592 & 0.111 & 0.320 \\
\hline M308 & 0.70581 & 33 & 236 & 0.70619 & 0.140 & 0.405 \\
\hline M314 & 0.70613 & 20 & 274 & 0.70633 & 0.073 & 0.211 \\
\hline M324 & 0.70601 & 16 & 278 & 0.70617 & 0.058 & 0.166 \\
\hline M331 & 0.70600 & 34 & 214 & 0.70643 & 0.159 & 0.460 \\
\hline M332 & 0.70593 & 12 & 299 & 0.70604 & 0.040 & 0.116 \\
\hline M339 & 0.70600 & 4 & 289 & 0.70604 & 0.014 & 0.040 \\
\hline
\end{tabular}

in most basalts of the Toranmal section, northwest of the study area $\left({ }^{87} \mathrm{Sr} /{ }^{86} \mathrm{Sr}=0.70467-0.70675\right.$; Mahoney et al 2000), and to the Poladpur Fm basalts (0.70573 - 0.70856; Lightfoot et al 1990), which seem to be widespread in the northern Deccan (cf. Mahoney et al 2000).

\section{Magmatic evolution}

The major and trace element variations observed in the central-western Deccan samples with more than $1.5 \mathrm{wt} \% \quad \mathrm{TiO}_{2}$ (figure 4 ) seem to be generally compatible with crystal fractionation processes starting from chemically similar parental magmas. Major element mass balance calculations indeed indicate that the transition from the most primitive basalts (D331, D325) to evolved basaltic andesites (D313, D310) can be obtained after fractionation (roughly 40-55\%) of olivine, plagioclase, clinopyroxene and minor oxides (table 5). Modelling of some elements incompatible with these phases (figure 4) also confirm that moderate degrees of fractionation (ca. 55\%) are needed in the transition from the most primitive basalt D333 to the most evolved basaltic andesites. It is worth noting that modelling was successful for both contamination- (and alteration) sensitive (e.g., $\mathrm{Ba}, \mathrm{K}_{2} \mathrm{O}$ ) and insensitive ( $\mathrm{Zr}, \mathrm{Nb}, \mathrm{Y}$, $\mathrm{TiO}_{2}$ ) elements and oxides, testifying that the fractionation stage recorded in these lavas occurred not too far from a closed system. The position of the samples in the pseudoternary diagram Forsterite-Diopside-Silica indicates that the evolution occurred at low pressure (close to the surface), and that the samples with pigeonite plot close or at the invariant point with olivine,
Table 5. Mass balance calculations. Two sets of partition coefficients were calculated: $D(N b)$ assume $D N b=0$, and hence $f=N b 0 / N b ; D(M B)$ assume $f$ (degree of residual liquid) of the mass balance calculations.

\begin{tabular}{|c|c|c|c|c|}
\hline & $\begin{array}{l}\text { From } \\
\text { D333 }\end{array}$ & $\begin{array}{c}\text { To } \\
\text { D313 }\end{array}$ & $\begin{array}{c}f(\mathrm{Nb}) \\
0.44 \\
\mathrm{D}(\mathrm{Nb})\end{array}$ & $\begin{array}{c}f(\mathrm{MB}) \\
0.48 \\
\mathrm{D}(\mathrm{MB})\end{array}$ \\
\hline $\mathrm{TiO}_{2}$ & 1.76 & 2.43 & 0.60 & 0.56 \\
\hline $\mathrm{P}_{2} \mathrm{O}_{5}$ & 0.17 & 0.30 & 0.31 & 0.23 \\
\hline $\mathrm{Sc}$ & 33 & 32 & 1.05 & 1.05 \\
\hline $\mathrm{V}$ & 335 & 435 & 0.68 & 0.64 \\
\hline $\mathrm{Cr}$ & 543 & 99 & 3.09 & 3.32 \\
\hline $\mathrm{Ni}$ & 126 & 56 & 2.00 & 2.11 \\
\hline $\mathrm{Zn}$ & 104 & 134 & 0.69 & 0.66 \\
\hline $\mathrm{Sr}$ & 204 & 262 & 0.69 & 0.66 \\
\hline $\mathrm{Y}$ & 24 & 45 & 0.23 & 0.15 \\
\hline $\mathrm{Zr}$ & 122 & 253 & 0.10 & 0.00 \\
\hline $\mathrm{Nb}$ & 7 & 16 & 0.00 & -0.11 \\
\hline $\mathrm{Ba}$ & 176 & 363 & 0.11 & 0.02 \\
\hline $\mathrm{La}$ & 12.54 & 27.73 & 0.03 & -0.08 \\
\hline $\mathrm{Ce}$ & 27.95 & 63.99 & -0.02 & -0.13 \\
\hline $\mathrm{Pr}$ & 3.74 & 8.65 & -0.03 & -0.14 \\
\hline $\mathrm{Nd}$ & 17.09 & 37.14 & 0.05 & -0.06 \\
\hline $\mathrm{Sm}$ & 4.21 & 8.79 & 0.10 & 0.00 \\
\hline $\mathrm{Eu}$ & 1.48 & 2.49 & 0.36 & 0.29 \\
\hline $\mathrm{Gd}$ & 4.98 & 9.04 & 0.27 & 0.19 \\
\hline $\mathrm{Tb}$ & 0.74 & 1.45 & 0.18 & 0.09 \\
\hline Dy & 4.18 & 8.67 & 0.10 & 0.00 \\
\hline Ho & 0.80 & 1.75 & 0.03 & -0.07 \\
\hline Er & 2.01 & 4.50 & 0.01 & -0.10 \\
\hline $\mathrm{Tm}$ & 0.30 & 0.63 & 0.08 & -0.02 \\
\hline $\mathrm{Yb}$ & 1.90 & 3.97 & 0.09 & -0.01 \\
\hline $\mathrm{Lu}$ & 0.28 & 0.55 & 0.15 & 0.05 \\
\hline
\end{tabular}

The transition between D333 and D313 was obtained through fractionation of $28.8 \%$ olivine, $38.3 \%$ cpx and $33 \%$ plagioclase.

plagioclase, clinopyroxene, pigeonite and liquid. Empirical whole-rock thermometers suitable for anhydrous tholeiitic compositions (e.g. Eggins 1992; Toplis and Carroll 1995) span temperatures from $1233-1227^{\circ} \mathrm{C}$ (D333 and D325) to 1125 $1123^{\circ} \mathrm{C}$, the latter obtained for the evolved basaltic andesites plotting close to or at the reaction point (D337 and D313; figure 6). Sano et al (2001) performed crystallization experiments on a composition very similar to the most primitive sample of this study (IG-02; $\mathrm{MgO}=9 \mathrm{wt} \%$; $\mathrm{TiO}_{2}=1.7 \mathrm{wt} \%$ ) and found olivine on the liquidus at $1220^{\circ} \mathrm{C}$, followed by augite at $1190^{\circ} \mathrm{C}$, plagioclase at $1170^{\circ} \mathrm{C}$, opaque oxides at $T<1180^{\circ} \mathrm{C}$ (depending on the oxygen buffer used in the experiments), and pigeonite at $1134^{\circ} \mathrm{C}$. The upper and lower temperature values closely match our empirical estimates. 


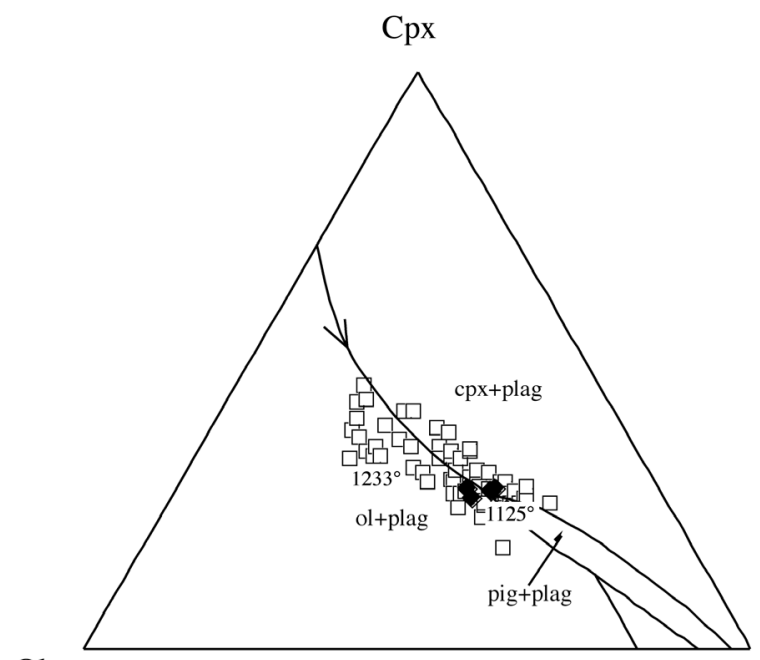

$\mathrm{Ol}$

Figure 6. One atmosphere pseudoternary diagram Forsterite-Diopside-Silica diagram (Grove et al 1982), with the rocks of central-western Deccan. The two temperatures shown in the diagram have been calculated for samples D333 and D313 using the $\mathrm{T}-\mathrm{MgO}$ relationship of Eggins (1992). Symbols as in figure 2 .

\section{Chemical correlations and petrogenesis}

The low-Ti lava flows from the surroundings of Dhule are chemically unrelated with the other central-western Deccan lava flows. On the other hand, they have very marked chemical similarities with:

- the low-Ti basalts and basaltic andesites widely cropping out in the Kathiawar peninsula, Gujarat (cf. Melluso et al 1995);

- the low-Ti dykes of the Tapti dyke swarm (Melluso et al 1999);

- the low-Ti basalt lavas cropping out at the Toranmal section (figure 8a; Mahoney et al 2000; Chandrasekharam et al 1999);

- the Bushe basalts (figure 8b).

The Dhule flows can be considered as the southernmost expressions of the Kathiawar low-Ti types. The low-Ti basalts and basaltic andesites, though being chemically evolved samples, have the lowest incompatible element contents. The pattern of the least incompatible elements and $\mathrm{Nb}$ is very similar to average enriched MORB (Sun and McDonough 1989), excluding the selective enrichment in $\mathrm{Rb}, \mathrm{Ba}, \mathrm{K}$ and light rare earth elements. The same is observed from the comparison of the rare earth element patterns (figure 5). This is an indication of their provenance from moderately incompatible element-poor mantle, similarly to the low-Ti basalts of Gujarat and the Tapti dykes (Melluso et al 1995, 1999). The flat heavy rare earth element patterns of these rocks indicate their ulti- mate provenance from sources located within the spinel stability field. The data also indicate that crustal contamination during the ascent to the surface, that may be the cause of the low strength element enrichment over $\mathrm{Nb}$, did not influence the heavy rare earth element contents appreciably. We finally note that contamination processes acting on these samples did not modify their normal low-pressure magmatic evolution, leaving them with compositions plotting on the low-pressure cotectics.

In the mantle normalized diagrams (figure 7), the central-western Deccan basalts have negative $\mathrm{Nb}, \mathrm{Sr}$ and $\mathrm{P}$ troughs. This feature makes these rocks more similar to lavas of lowermost formations of the Western Ghats (Khandala, Thakurvadi and Jawhar), to some of the Poladpur Fm. lava flows, and some high-Ti dykes of Tapti (Melluso et al 1999, and unpublished data) rather than typical Ambenali and Mahableshwar basalts (cf. figures 5 and 7) and highly enriched basalts of Kathiawar, Pavagadh and Rajpipla (Melluso et al 1995; Peng and Mahoney 1995).

The diagrams $\mathrm{Zr} / \mathrm{Y}$ vs. $\mathrm{TiO}_{2}$ (figure $8 \mathrm{~b}$ ) indicate that the samples with $\mathrm{TiO}_{2}>1.5 \mathrm{wt} \%$ plot in the same field of the Jawhar-Igatpuri Fm. and still broadly within the very scattered compositions of the Thakurvadi Fm. The Poladpur samples, at broadly the same $\mathrm{TiO}_{2}$, plot at slightly lower $\mathrm{Zr} / \mathrm{Y}$, but this may be the result of different analytical techniques involved. It is worth noting that, on the basis of radiogenic isotope data and multi-elemental statistics, basalts ascribed to the Poladpur Fm. have been found in the Toranmal section (Mahoney et al 2000).

The high values of the ratios between large ion lithophile elements and $\mathrm{Nb}$, together with the relatively high ${ }^{87} \mathrm{Sr} /{ }^{86} \mathrm{Sr}$ ratios, are evidence of a crustal input in all the central-western Deccan samples. We note that this feature is already present in the most mafic samples, and there is no evidence of increase of these ratios with increasing degree of fractionation. Therefore, this relative enrichment occurred in a very early stage of evolution (picrite basalt level, not represented in our sample set), or directly in the mantle source.

Concerning the chemical characteristics of mantle-derived magmas which generated the central-western Deccan sequence, from the range of $\mathrm{Zr} / \mathrm{Y}$ and $\mathrm{TiO}_{2}$ of primitive tholeiitic magmas (with $\mathrm{MgO}>9 \mathrm{wt} \%$ ) found throughout the province, the composition of the basalts of the central-western area, and fractionation models starting from different parental magmas (figure $8 \mathrm{~b}$ ), we note some features:

- The mafic parental magmas of the Deccan have a large range of variation of $\mathrm{Zr} / \mathrm{Y}$ and $\mathrm{TiO}_{2}$, with 


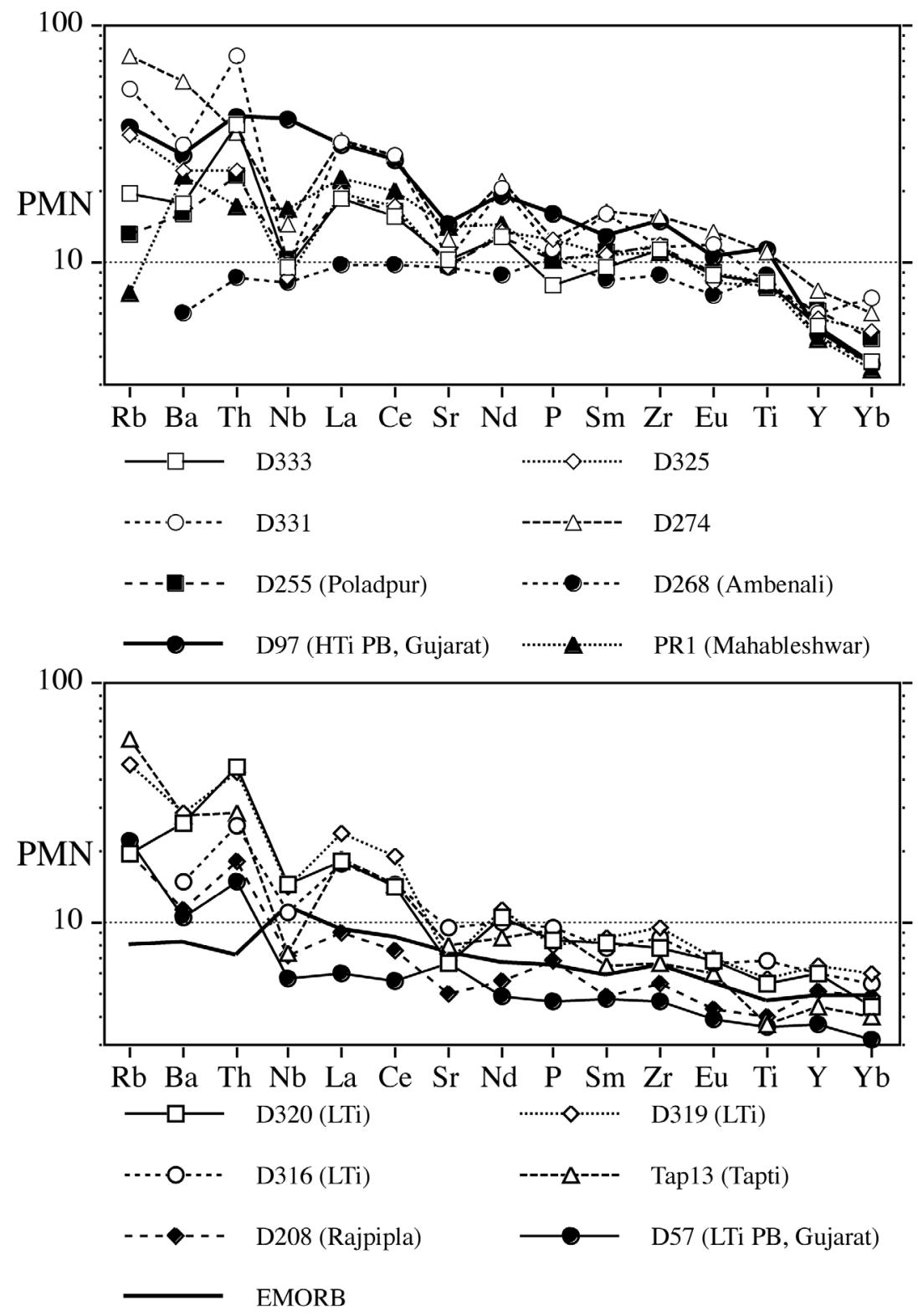

Figure 7. Primitive mantle-normalized incompatible (PMN) element diagrams for mafic rocks of southwestern Deccan, and Gujarat. Reference data are taken from Melluso et al 1995, 1999, and unpublished data. Primitive mantle values and E-MORB values of Sun and McDonough (1989).

the least enriched end-members being the low- $\mathrm{Ti}$ picrite basalts of Gujarat, and the most enriched end-members being the high-Ti picrite basalts of Pavagadh, and drillholes in Gujarat (Melluso et al 1995; Peng and Mahoney 1995; Krishnamurthy and Cox 1977).

- Fractional crystallization cannot be the cause of the range of $\mathrm{Zr} / \mathrm{Y}$ observed in the Deccan basalts and picrite basalts, thus implicating strong parental magma heterogeneity.

- The rocks of central-western Deccan with $\mathrm{TiO}_{2}>1.5$ wt.\% appear to have been generated by fractional crystallization starting from mildly enriched mafic precursors, whereas the low-Ti basalts may have had incompatible element-depleted primitive precursors, such as the picrite basalts of Gujarat (Melluso et al 1995). These latter resemble mid-ocean ridge basalts when high field strength elements are considered.

- A mixing curve between the low-Ti and highTi basalts of Gujarat is roughly able to account for HFSE variations of the likely mantle-derived magmas throughout the Deccan. This can indicate that both high field strength elementdepleted and -enriched sources were involved, to variable mixing proportions, in the melting processes of the mantle beneath the province. This has to be verified on a larger data set. 

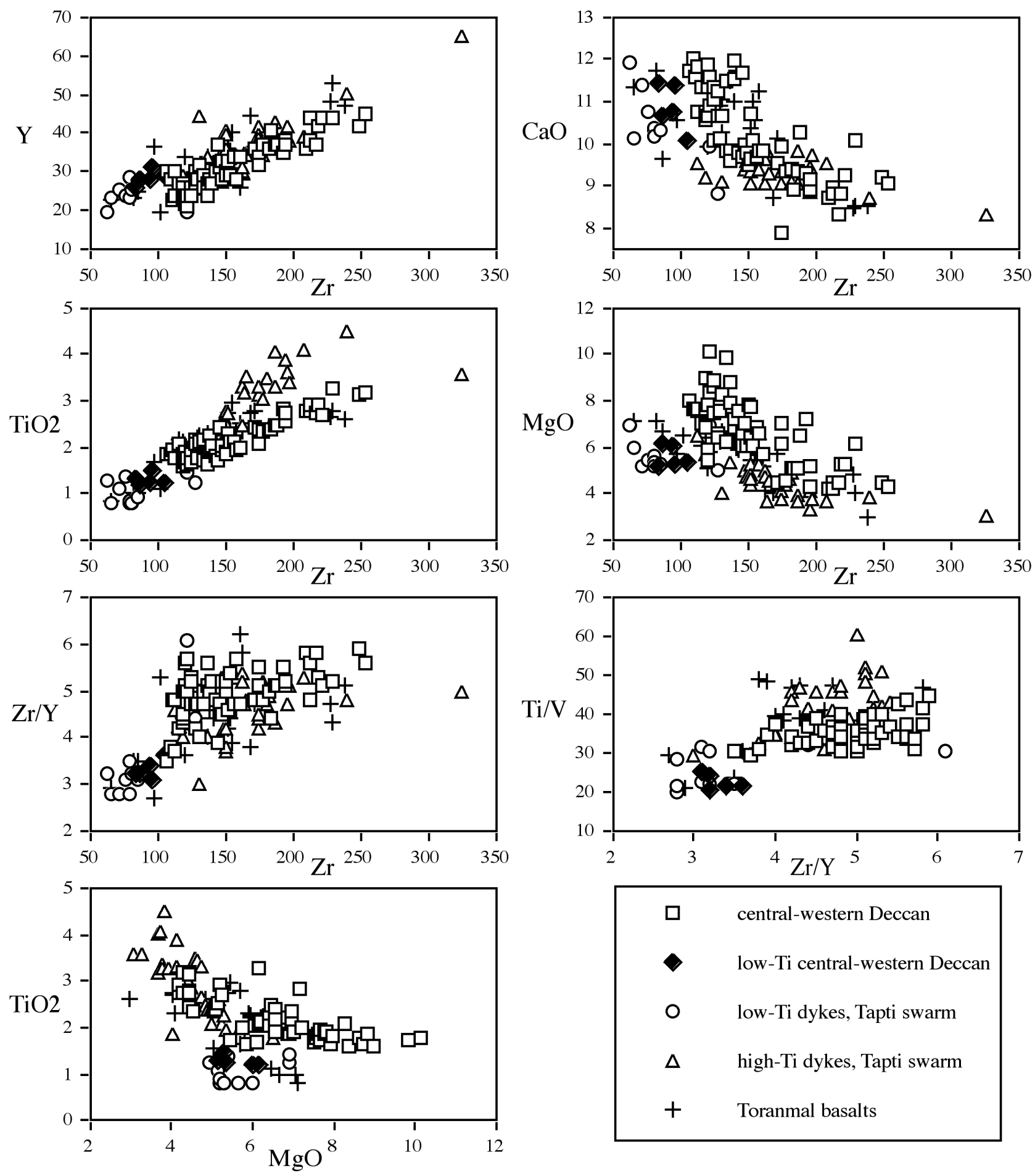

Figure 8(a and $\mathbf{b})$. Chemical correlation diagrams between the central western Deccan basalts and other Deccan samples. The chemical data on the Toranmal section are from Mahoney et al (2000); the chemical data on the Tapti dykes are taken from Melluso et al (1999), and for the Gujarat basalts are taken from Melluso et al (1995). Main references for other basalt data are: Bodas et al 1988; Khadri et al 1988; Cox and Hawkesworth 1985; Lightfoot and Hawkesworth 1988; Lightfoot et al 1990, Peng et al 1994 and other references therein.

\section{Concluding remarks}

This study on the flood basalt sequence of centralwestern Deccan has the following implications for the knowledge of this province:
- The most mafic lava flows of this area have been found in the Western Ghats slopes (close to Igatpuri), whereas the most evolved flows have been mostly found in the eastern-most areas. This may be the result of a temporal change of 

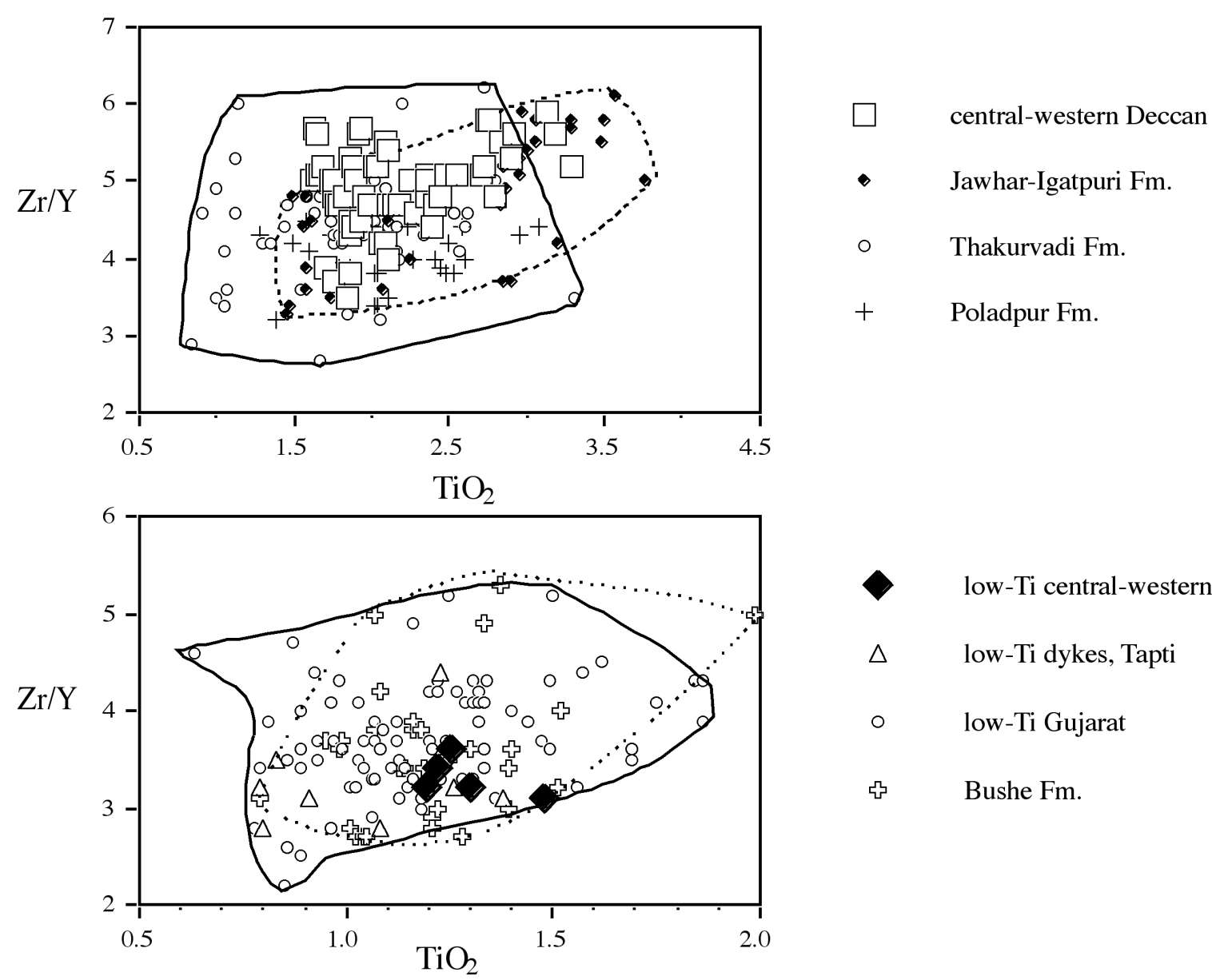
low-Ti central-western Deccan
$\triangle \quad$ low-Ti dykes, Tapti
○ low-Ti Gujarat
њ Bushe Fm.
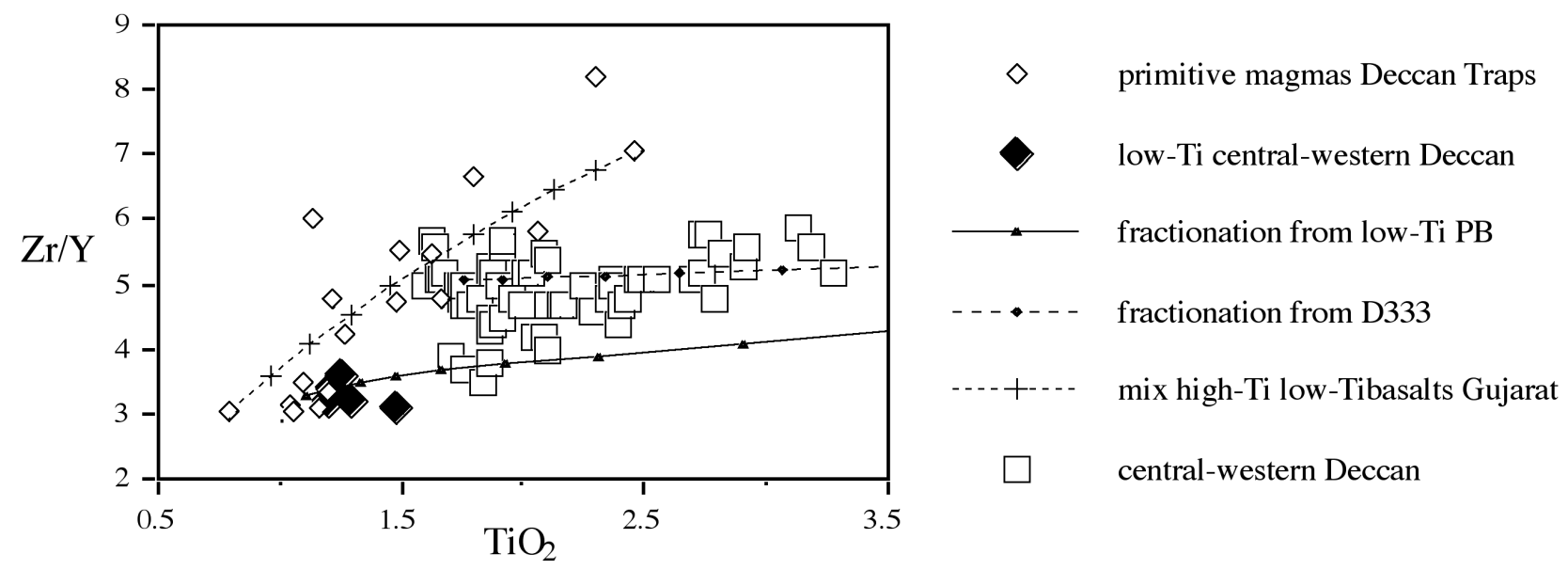

Figure 8(b). (Continued)

chemistry of the erupted magmas (more mafic liquids erupted earlier, evolved liquids later and upward sequence, and preserved only far from this sector of the province).

- Most samples have major and trace element compositional trends typical of low-pressure liquid lines of descent of tholeiitic magmas, caused by olivine, clinopyroxene and plagioclase fractionation, starting from the most mafic liquids of this study.
- The incompatible element contents and ratios of the most mafic rocks and the Sr-isotope composition of the central Deccan samples indicate a crustal input. However, the evolution from basalt to basaltic andesite most likely took place in a substantially closed system.

- The lava flows have a strong resemblance to some chemical types of the lowermost formations of the Deccan, and probably also with the Poladpur basalts. The Dhule lava flows with low-Ti 
affinity are close analogues of the low-Ti rocks widespread in the Kathiawar peninsula and in the Tapti swarm (cf. Melluso et al 1995, 1999; Mahoney et al 2000), and should be referred to as a discrete magma type, unlike the low-Ti rocks of the Bushe Fm. This magma type can also be distributed as a chemical type in the lowermost formations.

\section{Acknowledgements}

We wish to thank M. Serracino for his skilled help in obtaining microprobe analyses, A Canzanella for $\mathrm{XRF}$ determinations and $\mathrm{R}$ Tassinari for ICP-MS determinations. Peter Hooper is thanked for allowing intercalibration between Naples and Washington State University XRF data and for several discussions and advice at various stages, and P Brotzu, V Morra and K V Subbarao are thanked for their advice. The official review of Hetu Sheth was much appreciated. This work has been supported by MURST and MIUR (COFIN) grants to L Beccaluva, P Brotzu and L Melluso.

\section{Appendix: Analytical techniques}

Mineral compositions were obtained on polished thin sections using a CAMECA electron microprobe at CNR-C.S.Q.E.A. Rome. Silicates and oxides were used as standards. The data were reduced utilizing the CAMECA's PAP correction method.

Sample chips, obtained from a steel jaw crusher, were first washed in distilled water and then ground in a low-blank agate mortar. Major and trace elements were analysed at Napoli (CISAG) with a Philips PW1400 X-Ray fluorescence (XRF) spectrometer (more details in Melluso et al 1995, 2001). Precision is estimated to be within $1 \%$ for $\mathrm{SiO}_{2}$, $\mathrm{TiO}_{2}, \mathrm{Al}_{2} \mathrm{O}_{3}, \mathrm{Fe}_{2} \mathrm{O}_{3 \mathrm{t}}$ and $\mathrm{CaO}$, better than $6 \%$ for $\mathrm{K}_{2} \mathrm{O}, \pm 0.03 \mathrm{wt} \%$ for $\mathrm{MnO}$ and $\mathrm{P}_{2} \mathrm{O}_{5}$, generally better than 5-10\% for Sc, V, Cr, Ni, Cu, Ba, Zn, Sr, Y and $\mathrm{Zr}$ in the observed compositional ranges, and $\pm 1 \mathrm{ppm}$ for $\mathrm{Rb}$ and $\mathrm{Nb}$ below $10 \mathrm{ppm}$. $\mathrm{Na}$ and $\mathrm{Mg}$ were analysed by atomic absorption spectrophotometry (AAS) at Napoli. Typical precision is better than $2 \%$ for $\mathrm{Mg}$ and better than $6 \%$ for $\mathrm{Na}$.

In order to fully compare XRF data from different laboratories, cross-checking of samples analysed at Naples and Washington State University (courtesy of Peter Hooper) was performed. Bias has been observed for some $\mathrm{P}$ determinations and for $\mathrm{Ba}$, though the significance is uncertain, whereas absolutely comparable values have been obtained for the other elemental determinations, in particular for the key incompatible elements Zr, Y,
$\mathrm{Nb}$ and Ti. Therefore, major emphasis was given to the elements with comparable analytical uncertainty and accuracy, in order to make all the inferences useful to general readership.

Rare earth elements, U, Th, Pb, Ta and Hf were analysed for a subset of samples by inductively coupled plasma-mass spectrometry (ICP-MS) at C.R.P.G. Nancy (France) (samples D293, D316, D333, D335), and Ferrara University (Italy) (samples D274, D288, D294, D313, D319, D320, D325, D330, D331, D339; table 3). Uncertainty in the measurements are typically within $5-10 \%$ relative for most elements in the observed compositional ranges.

Strontium isotopic compositions have been analyzed at Rome University, using a VG54E mass spectrometer. Full details of the analytical procedures are described in Comin-Chiaramonti et al (1997).

\section{References}

Beane J E and Hooper P R 1988 A note on the picrite basalts from the western Ghats, Deccan Traps, India; Mem. Geol. Soc. India 10 117-133

Bodas M S, Khadri S F R and Subbarao K V 1988 Stratigraphy of the Jawhar and Igatpuri Formations, Western Ghats lava pile, India; Mem. Geol. Soc. India 10 235-252

Boynton W B 1984 Cosmochemistry of Rare Earth Elements: Meteorite Studies. In: Henderson P (ed.): Rare Earth Element Geochemistry, Elsevier, Amsterdam, pp. $63-114$

Chandrasekharam D, Mahoney J J, Sheth H C and Duncan R A 1999 Elemental and Nd-Sr-Pb isotope geochemistry of flows and dikes from the Tapi rift, Deccan flood basalt province, India; J. Volcanol. Geotherm. Res. 93 111-123

Comin-Chiaramonti P, Cundari A, Piccirillo E M, Gomes C B, Castorina F, Censi P, De Min A, Marzoli A, Speziale S and Velazquez V F 1997 Potassic and sodic igneous rocks from eastern Paraguay: their origin from the lithospheric mantle and genetic relationships with the associated Paranà flood tholeiites; J. Petrol. 38 $495-528$

Cox K G and Hawkesworth C J 1985 Geochemical stratigraphy of the Deccan Traps at Mahabaleshwar, Western Ghats, India, with implication for open system processes; J. Petrol. 26 355-387

Eggins S M 1992 Origin and differentiation of picritic arc magmas, Ambae (Aoba), Vanuatu; Contrib. Mineral. Petrol. 114 79-100

Grove T L, Gerlach D C and Sando T W 1982 Origin of calcalkaline series lavas at Medicine Lake Volcano by fractionation, assimilation and mixing; Contrib. Mineral. Petrol. 80 160-182

Hooper P R 1994 Sources of continental flood basalts: the lithospheric component; In: Subbarao K V (ed.) Volcanism, Wiley Eastern Ltd. pp 29-53

Hooper P R 1999 The Deccan Traps: a personal perspective; In: Subbarao K V (ed.) Deccan Volcanic Province. W D West Volume; Mem. Geol. Soc. India 43 153-165

Khadri S F R, Subbarao K V, Hooper P R and Walsh J N 1988 Stratigraphy of Thakurvadi Formation, Western 
Deccan Basalt Province, India; Mem. Geol. Soc. India 10 280-304

Khadri S F R, Subbarao K V and Walsh J N 1999a Stratigraphy, form and structure of the east Pune basalts, western Deccan basalt province, India; In: Subbarao K V (ed.) Deccan Volcanic Province. W D West Volume; Mem. Geol. Soc. India 43 179-202

Khadri S F R, Walsh J N and Subbarao K V 1999b Chemical and magneto-stratigraphy of Malwa Traps around Magraba region, Dhar district (Madhya Pradesh); In: Subbarao K V (ed.) Deccan Volcanic Province. W D West Volume; Mem. Geol. Soc. India 43 203-218

Krishnamurthy P and Cox K G 1977 Picrite basalts and related lavas from the Deccan Traps of Western India; Contrib. Mineral. Petrol. 62 53-75

Krishnamurthy P and Cox K G 1980 A potassium-rich alkalic suite from the Deccan Traps, Rajpipla, India; Contrib. Mineral. Petrol. 73 179-189

Lightfoot P C and Hawkesworth C J 1988 Origin of Deccan Trap lavas: evidence from combined trace element and Sr-, Nd- and Pb-isotope data; Earth Planet. Sci. Lett. 91 89-104

Lightfoot P C, Hawkesworth C J, Devey C W, Rogers N W and Van Calsteren P W C 1990 Source and differentiation of Deccan Trap lavas: implications of geochemical and mineral chemical variations; J. Petrol. 31 $1165-1200$

Mahoney J J, Macdougall J D, Lugmair G W, Gopalan K and Krishnamurthy P 1985 Origin of contemporaneous tholeiitic and K-rich alkalic lavas: a case study from the northern Deccan Plateau, India; Earth Planet. Sci. Lett. 72 39-53

Mahoney J J 1988 Deccan Traps; In: Macdougall J D (ed.) "Flood Basalts" Kluwer, Dordrecht pp. 151-194

Mahoney J J, Sheth H C, Chandrasekharam D and Peng Z X 2000. Geochemistry of flood basalts of the Toranmal section, northern Deccan Traps, India: implications for regional Deccan stratigraphy; J. Petrol. 41 1099-1120

Mahoney J J, Duncan R A, Khan W, Gnos E and McCormick G R 2002 Cretaceous volcanic rocks of the South Tethyan suture zone, Pakistan: implications for the Réunion hotspot and Deccan Traps; Earth Planet. Sci. Lett. 203 295-310

Melluso L, Beccaluva L, Brotzu P, Gregnanin A, Gupta A K, Morbidelli L and Traversa G 1995 Constraints on the mantle sources of the Deccan Traps from the petrology and geochemistry of the basalts of Gujarat State (Western India); J. Petrol. 36 1393-1432

Melluso L, Sethna S F, Morra V, Khateeb A and Javeri P 1999 Petrology of the mafic dyke swarm of the Tapti river in the Nandurbar area (Deccan volcanic province); In: Subbarao K V (ed.) Deccan Volcanic Province. W D West Volume; Mem. Geol. Soc. India 43 735-756

Melluso L, Morra V, Brotzu P and Mahoney J J 2001 The Cretaceous igneous province of central-western Madagascar: evidence for heterogeneous mantle sources, crystal fractionation and crustal contamination; J. Petrol. 42 $1249-1278$
Mitchell C and Widdowson M 1991 A geological map of the southern Deccan Traps, India and its structural implications; J. Geol. Soc. London 148 495-505

Najafi S J, Cox K G and Sukheswala R N 1981 Geology and geochemistry of the basalt flows (Deccan Traps) of the Mahad-Mahableshwar section, India; In: Subbarao K V and Sukheswala R N (eds.) Deccan Volcanism; Mem. Geol. Soc. India 3 300-315

Pande K 2002 Age and duration of the Deccan Traps, India: a review of radiometric and paleomagnetic constraints; Proc. Indian Acad. Sci. 111 115-123

Peng Z X and Mahoney J J 1995 Drillhole lavas in the Deccan Traps and the evolution of the Reunion plume; Earth Planet. Sci. Lett. 134 169-185

Peng Z X, Mahoney J J, Hooper P R, Harris C and Beane J E 1994 A role for lower continental crust in flood basalt genesis? Isotopic and incompatible element study of the lower six formations of the Western Deccan Traps; Geochim. Cosmochim. Acta $\mathbf{5 8}$ 267-288

Peng Z X, Mahoney J J, Hooper P R, Macdougall J D and Krishnamurthy P 1998 Basalts of the northeastern Deccan Traps, India: isotopic and elemental geochemistry and relation to southwestern Deccan stratigraphy; J. Geophys. Res. 103 29843-29865

Sano T, Fujii T, Deshmukh S S, Fukuoka T and Aramaki S 2001 Differentiation processes of Deccan Trap basalts: contribution from geochemistry and experimental petrology; J. Petrol. 42 2175-2195

Sen G 2001 Generation of Deccan Trap magmas; Proc. Indian Acad. Sci. 111 409-431

Sheth H C, Mahoney J J and Chandrasekharam D 2004 Geochemical stratigraphy of Deccan flood basalts of the Bijasan Ghat section, Satpura Range, India; J. Asian Earth Sci. 23 127-139

Subbarao K V, Chandrasekharam D, Navaneethakrishnan P and Hooper P R 1994 Stratigraphy and structure of parts of the central Deccan basalt province: eruptive models; In: Subbarao K V (ed.) Volcanism, Wiley Eastern Ltd. pp 321-332

Subbarao K V and Hooper P R 1988 Reconnaissance map of the Deccan Basalt Group in the Western Ghats, India; Mem. Geol. Soc. India $\mathbf{1 0}$

Sun S-s and McDonough W F 1989 Chemical and isotopic systematics of oceanic basalts: implications for mantle composition and processes; In: Saunders A D and Norry M J (eds) Magmatism in the Ocean Basins. Geol. Soc. Spec. Publ. 42 313-345

Talusani R V R 2001 A newly reported alkali basalt flow near Bhir, Deccan Volcanic Province, India; J. Asian Earth Sci. 19 501-506

Toplis M J and Carroll M R 1995 An experimental study of the influence of oxygen fugacity on Fe-Ti oxide stability, phase relations, and mineral-melt equilibria in ferrobasaltic systems; J. Petrol. 36 1137-1170

Widdowson M, Pringle M S and Fernandez O A 2000 A post K-T boundary (Early Paleocene) age for Deccantype feeder dykes, Goa, India; J. Petrol. 41 1177-1194 